\title{
VII. Nach der Rückkehr ins Parlament $1928-1930$
}

Als Paul Reynaud am 29. Januar 1928 zum ersten Mal mit dem neuen radikalsozialistischen Parteichef Edouard Daladier in öffentlichem Streitgespräch die Klingen kreuzte ${ }^{1}$, standen sich zwei Politiker gegenüber, die gleichermaßen einer „Scharniergeneration“ angehörten. 1878 bzw. 1884 geboren, hatten beide die Frühphase der Republik, den Höhepunkt der Dreyfus-Affäre und die Abwehr republikfeindlicher Strömungen von rechts als bereits erwachsene Männer erlebt. Politisch waren sie verwurzelt in dieser Schlüsselphase der Konstituierung des republikanischen Konsensus und gelangten doch noch jung genug an die Schwelle echter und vielversprechender politischer Wirksamkeit, um mit angewachsener Erfahrung den Anspruch auf politische Reform noch nicht beiseitegelegt zu haben. Hoffnungsträger ihrer Generation im Hinblick auf die Bewältigung der neuartigen Probleme der Nachkriegszeit, waren gegen Ende der zwanziger Jahre beide Politiker geworden: nach glänzender Parteikarriere, die innerhalb weniger Jahre aus „zweiter Reihe“ in Schlüsselpositionen auf parteipolitischer und staatlicher Ebene geführt hatte, der eine; nach parteipolitisch abgestützter, doch insgesamt weniger durch sie gebundener Karriere am Rande des Bloc national, dann als zunehmend bedeutender Sprecher der bürgerlichen Opposition, der andere. Begünstigt durch die allgemeine wie die parteiinterne politische Entwicklung hatte sich freilich Daladier bereits einen „Karrierevorsprung“ erarbeitet. Dabei waren die Ausgangspositionen auf den ersten Blick durchaus vergleichbar gewesen. Wie Reynaud war auch Daladier als Befürworter der "Loi de trois ans“ im Wahlkampf von 1914 gescheitert und erst 1919, geprägt vom Erlebnis des Weltkriegs, zum erstenmal in die Kammer der Abgeordneten eingezogen. Dann aber hatte es der Protegé Herriots ab Juni 1924 bereits mehrfach zum Minister in den Kabinetten Herriot, Painlevé und Briand gebracht, bevor er als Vertreter einer neuen Generation Ende 1927 an die Spitze des „Parti républicain, radical et radical-socialiste“ gewählt worden war. ${ }^{2}$

Daladier und Reynaud verkörperten in geradezu idealtypischer Weise Varianten von Werdegängen im liberalen Milieu, die ihren Ausgang in sehr verschiedener sozialer Herkunft genommen und die mit sehr unterschiedlichen politischen Temperamenten begabten Politiker bei allen äußeren Berührungspunkten bis 1927 an

1 Vgl. dazu den Bericht „La propagande de l'Alliance. A Poitiers. M. Paul Reynaud fait le procès du cartellisme“ sowie die Erläuterungen Reynauds: „La vérité sur la Conférence contradictoire de Poitiers. Les questions de Paul Reynaud à M. Edouard Daladier", in: La République Démocratique, 5. 2. 1928.

2 Zur Person Edouard Daladiers grundlegend: Elisabeth du Réau, Edouard Daladier. 1884-1970, Paris 1993; Berstein, Parti Radical II, S. 45-50. 
entgegengesetzte Pole der politischen Mitte geführt hatten. So trat der aus einfachen Verhältnissen stammende Bäckerssohn und Gymnasiallehrer Daladier, dessen wortkarges, ja als mürrisch empfundenes Wesen ihm in Verbindung mit seinem bulligen Äußeren den Ruf entschlossener Energie eingetragen hatte, als Exponent einer linken Reformströmung seiner Partei ins neue Amt. Der ebenso hartnäckige, doch geschmeidigere, dialektisch geschulte, zu eloquenter Selbstdarstellung neigende Industriellensohn und Anwalt Reynaud auf der anderen Seite kehrte dagegen 1928 als Vertreter des rechten Flügels der "Alliance“ ins Parlament zurück. ${ }^{3}$ Die in dieser Konstellation sich andeutenden unterschiedlichen politischen Standpunkte konkretisierten sich im Verlauf der dreißiger Jahre in diversen ernsteren Meinungsverschiedenheiten zwischen den beiden Politikern. Das daraus erwachsene, durch persönlich begründete Animositäten zusätzlich belastete Verhältnis verhinderte nicht, daß beide nach der Rechtswendung Daladiers im Herbst 1938 zur Zusammenarbeit unter dem Vorzeichen der „Défense nationale“ fanden; erst im Umfeld der Ministerpräsidentschaft Reynauds erfolgte der endgültige Bruch.

$\mathrm{Zu}$ Beginn der 14. Legislaturperiode freilich beeinflußten sich beider politische Wirkungskreise nur indirekt. Daladier war in die Wahlen vom April 1928 mit dem erklärten Programm gegangen, dem unklaren Verhältnis seiner Partei zur „Union nationale" ein Ende zu bereiten und gezielt auf eine Zusammenarbeit mit den Sozialisten unter dem Vorzeichen der „Union des Gauches“ zuzusteuern. Es dauerte dann allerdings noch bis zu der Linkswendung des Kongresses von Angers im Oktober 1928 und der Klärung der Kräfteverhältnisse in der Kammer im Januar 1929, bis die radikalsozialistische Partei unter seiner Führung endgültig in die Opposition zur Regierung Poincaré trat. Als Folge dieser Wendung wurde Daladier mit starker innerparteilicher Gegnerschaft seitens der Anhänger einer Koalition nach rechts konfrontiert und konnte die Ablösung als Parteichef zugunsten seines zum Rivalen gewordenen Mentors Herriot nur mit Mühe abwenden. Daß er dann auch die erste Chance seiner politischen Laufbahn zur Regierungsbildung im Oktober 1929 aufgrund des Unwillens der vergeblich umworbenen Sozialisten nicht nutzen konnte, besiegelte das endgültige Scheitern seines seit 1927 in Angriff genommenen Linksexperiments. Wie bei Reynaud die Erfahrungen aus dem Wahlkampf von 1926 maßgeblich geworden waren für seine Skepsis gegenüber einem Wahlbündnis mit der republikanischen Linken, so wirkte das Erlebnis des „sozialistischen Verrats" vom Herbst 1929 prägend auf Daladier, der fortan wie die übrige radikalsozialistische Führung einem Bündnis mit den Sozialisten zutiefst mißtrauisch gegenüberstand. ${ }^{4}$ Die Niederlage des Parteichefs barg daneben in sich bereits den Keim für die Rückkehr eines traditionelleren, auf wahlpolitische Unabhängigkeit bedachten Radikalismus in die Führungsspitze. So bedeutete die Wiederwahl Herriots als Parteichef im September 1931 nurmehr die formale Be-

3 So die Einschätzung der Sûreté in ihrem Bericht „A. 2877. A/s d'un congrès de l'Alliance démocratique et du Parti Radical-socialiste" vom 3.12. 1927 (AN, F¹3962). Zusammen mit Barthélemy stellte ihn die Analyse einem Linksflügel um Flandin, François-Poncet und Le Trocquer gegenüber.

4 Zur Entwicklung der radikalsozialistischen Partei und ihres Chefs Daladier zwischen dem Kongreß von Angers und Herbst 1929 vgl. Berstein, Parti Radical II, S. 70-151. 
stätigung seines Status als „président sans titre“, den er angesichts des rapiden Machtverlusts Daladiers de facto bereits seit Sommer 1930 innehatte. $^{5}$

Für Paul Reynaud dagegen leitete die seit den Wahlen von 1928 einsetzende und mit dem Austritt der radikalsozialistischen Minister aus der Regierung Poincaré konsolidierte Rechtsverschiebung der parlamentarischen Mehrheit ${ }^{6}$ eine neue Etappe seiner politischen Karriere ein. Der Wiederaufstieg der „modérés“ zur dominierenden Gruppe in der Regierungsmehrheit eröffnete ihm neue parlamentarische Handlungsmöglichkeiten, die er nutzte, um sich bis Sommer 1929 als einer ihrer Führer zu profilieren. Die vorher so favorisierten sozialpolitischen Fragen spielten dabei in seiner Politik keine Rolle. Vielmehr bot sich die Gelegenheit im Rahmen einer inner- wie außerparlamentarischen Debatte, die eines der wichtigsten Probleme der französischen Außenpolitik seit Kriegsende, in zeitgenössischer Einschätzung „eine der bedeutendsten Debatten der Dritten Republik" " betraf: die Frage der Regelung der "dettes interalliées“.

\section{1. „Le petit caporal de la majorité“. 8 Paul Reynaud und die Ratifizierung der interalliierten Schulden im Juli 1929 - Karrierewege im liberalen Milieu}

Bei Beginn des Jahres 1926 zählte Frankreich zu den letzten europäischen Staaten, die noch keine Vereinbarung zur Konsolidierung ihrer während und nach dem Ersten Weltkrieg gegenüber den Vereinigten Staaten entstandenen Schulden abgeschlossen hatten. ${ }^{9}$ Die französischen Verpflichtungen umfaßten eine "politische“, d.h. im Zuge amerikanischer Hilfeleistungen an das kriegführende Frankreich entstandene, und eine rein „kommerzielle“ Schuld. Sie resultierten einerseits aus Krediten, die die USA zum Kauf von Rüstungsgütern auf dem amerikanischen Markt und - nach dem Waffenstillstand - als Wiederaufbauhilfen gewährt hatten; andererseits beinhalteten sie jene Verbindlichkeiten, die Frankreich durch die Übernahme größerer Mengen von Kriegsmaterial eingegangen war, das die amerikanischen Truppen in Europa zurückgelassen hatten. Der Gesamtwert dieser Verpflichtungen belief sich bis Juni 1925 auf etwa 4,2 Milliarden Dollar. ${ }^{10}$ Erst am

Ebenda, S. 151-185.

Maycur, Troisième République, S. 287-290.

"La ratification des accords", in: Le Temps, 22. 7. 1929.

Paris-Midi, 7. 6. 1929.

9 Zum Verlauf des diplomatischen Prozesses um die Regelung der Schuldenfrage: Denise Artaud, La question des dettes interalliées et la reconstruction de l'Europe (1917-1929). 2 Bände, Paris 1978; Dies., Die Hintergründe der Ruhrbesetzung 1923. Das Problem der interalliierten Schulden, in: VfZ 27 (1979), S. 241-259; Dies., La question des dettes interalliées et la reconstruction de l'Europe, in: Revue Historique 261 (1979), S. 363-382; Ellen Schrecker, The Hired Money. The French Debt to the United States, 1917-1929, New York 1978. Als faktenreiche Analyse ist auch für dieses Thema die Darstellung von Weill-Raynal immer noch unersetzlich, hier: Ders., Réparations III, S. 365-392; daneben: Robert Lacour-Gayet, Le problème de la dette française envers les EtatsUnis après la Première Guerre mondiale (1917-1932), in: Revue d'histoire diplomatique 75 (1961), S. 10-24; als aufschlußreiche zcitgenössische Darstellung: Lucien Petit, Histoire des finances extérieures de la France. Le règlement des dettes interalliées, 1919-1929, Paris 1932.

10 Die genauen Summen beliefen sich bezüglich der "politischen“ Schuld auf 2,933 Mrd. Dollar, zu- 
29. April 1926 wurde in Washington eine amerikanisch-französische Vereinbarung unterzeichnet, worin die rein kommerzielle, also a priori in jedem Falle voll rückzuerstattende Schuld mit der „politischen“ Kriegsschuld gleichgesetzt wurde. Für die französische Seite bedeutete der „Accord Mellon-Bérenger“ insofern einen Gewinn, als erstere nicht, wie ursprünglich vorgesehen, nach zehnjähriger Laufzeit zum 1. August 1929 beglichen werden mußte, sondern gemäß den günstigeren Zinssätzen und der Verteilung auf 62 Annuitäten, die für letztere galten, behandelt wurde. Am 12. Juli $1926 \mathrm{kam}$ in London eine ähnlich gestaltete Vereinbarung mit Großbritannien zur Unterzeichnung. ${ }^{11}$

Bis Sommer 1929 freilich hatten Parlament und Regierung Poincaré die Ratifizierung immer wieder hinausgeschoben, wofür ein eng verflochtenes Bündel von innenpolitischen, binnen- wie außenwirtschaftlichen und reparationspolitischen Faktoren maßgeblich geworden war.

Der neue Ministerpräsident selbst hatte sich wenige Tage vor seiner Berufung am 14. Juli 1926 gegen die Annahme der Verträge in unmodifizierter Form ausgesprochen und dabei jene Argumente wiederaufgegriffen, die in einer ersten Kammerdebatte zu den Washingtoner Verträgen zwischen dem 6. und 9. Juli 1926 von den Ratifizierungsgegnern ins Feld geführt worden waren: die Gefahr einerseits, daß bei einer möglichen Kommerzialisierung der französischen Schuld Obligationen in die Hand auch deutscher Gläubiger geraten konnten, die damit ein willkommenes Druckmittel zur Revision des Versailler Systems gewännen; das Fehlen von Vorbehaltsklauseln andererseits, die Vorsorge für den Fall einer Einschränkung der französischen Zahlungsfähigkeit träfen, insbesondere beim Ausbleiben deutscher Reparationsleistungen. ${ }^{12}$ Unklar blieben allerdings die Art und Tragweite der Modifikationen, die die neue Regierung der Kammer empfehlen und gegenüber den Gläubigern vertreten würde. Die einfache Ratifizierung, sei sie auch verbrämt durch juristisch unerhebliche, erläuternde "considérants“, die als Kompromißmöglichkeit ins Auge gefaßt worden waren, konnte bei der ablehnenden Stimmungslage in der Kammer die Existenz der Regierung gefährden. Eine klare Ablehnung oder auch mit Änderungsanträgen in Form von „amendements“ versehene Zustimmung dagegen barg nach ersten offiziellen Reaktionen aus den USA die Gewißheit der sofortigen Fälligkeit der kommerziellen Schuld, die Wiederaufnahme der Verhandlungen über den politischen Teil und das Risiko einer nachfolgenden Verschärfung der Zahlungsbedingungen in sich. Poincaré setzte vorerst auf Zeitgewinn. ${ }^{13}$

Dabei hatten die rein finanzpolitischen Problemlagen, die sich ursprünglich einer Ratifikation entgegengestellt hatten, seit 1926 bedeutend an Gewicht verloren. Mit der faktischen Konsolidierung des Staatshaushalts durch Poincaré, der massiven Rückkehr von Devisen und Goldbeständen nach Frankreich und der Stabilisierung des Franc ab Juli 1926 war eine Schuldenregelung rein finanztechnisch

züglich 881 Mio. Dollar Zinsschuld am 15. Juni 1925, für den „kommerziellen“ Teil auf 407 Mio. Dollar (Weill-Raynal, Réparations III, S. 377 f.).

1 Artaud, Dettes interalliées, S. 774-807; Schrecker, Hired Money, S. 219-234, 344-366.

$12 \mathrm{Zu}$ den Äußerungen Poincarés in der spanischen Tageszeitung Vanguardia vom 14. 7. $1926 \mathrm{vgl}$. Weill-Raynal, Réparations III, S. $394 \mathrm{f}$.

13 Ebenda, S. 396. 
sehr viel mehr in den Bereich des Möglichen getreten, als das noch unmittelbar nach Kriegsende oder selbst im Frühjahr 1926 der Fall gewesen war. Dennoch hielt die französische Führung weiterhin an ihrem Grundsatz vom Vorrang des Erhalts deutscher Zahlungen vor der Begleichung der eigenen Schulden fest, der seit 1919 das diplomatische Vorgehen bestimmt und die öffentliche Meinung im Land tief geprägt hatte. ${ }^{14}$

Ausschlaggebend für die über beinahe drei Jahre fortgesetzte Verzögerungstaktik war deshalb vielmehr, daß sich erst im Frühsommer 1929 eine befriedigende Lösung zur Koordinierung der interalliierten Rückzahlungen mit deutschen Reparationsleistungen abzeichnete. ${ }^{15}$ Die Idee der Verknüpfung beider Komplexe war bereits während der Versailler Friedensverhandlungen seitens der englischen Regierung angesprochen und zu Beginn der zwanziger Jahre zu einem Konzept ausgebaut worden, in dem sich die Annullierung der gegenseitigen Verpflichtungen mit der drastischen Reduzierung der deutschen Schuld verbinden sollte. Ebenso wie ein Plan des französischen Finanzministeriums vom Januar 1922, der die Verwendung der Bons der sogenannten C-Obligationen des Londoner Schuldenabkommens von 1921 zur Begleichung der interalliierten Verbindlichkeiten Frankreichs vorsah, trafen alle derartigen Vorschläge auf die hartnäckige Ablehnung der USA. Das wachsende Zerwürfnis Poincarés mit Großbritannien, das sich mit der Balfour-Note vom August 1922 den französischen Plänen auf Bildung einer gemeinsamen europäischen Schuldnerfront gegenüber den USA entzogen hatte und ins "Gläubigerlager" übergewechselt war, trug seinerseits zur Verzögerung einer Klärung bei. ${ }^{16}$ Der Dawes-Plan vom April 1924 schließlich war zwar von seinen Urhebern auch als Rahmen zur Lösung der Schuldenfrage gedacht. Da er aber nach wie vor die Zahl der durch Deutschland zu leistenden Annuitäten nicht festlegte, erwies er sich als ungeeignete Basis für eine daran orientierte interalliierte Schuldenvereinbarung. ${ }^{17} \mathrm{Da}$ die Arbeit des im Dezember 1928 neuerlich einberufenen Expertengremiums zur Erstellung eines Zahlungsplans im wesentlichen eben der Beseitigung dieses Mangels und der Festlegung neuer Annuitäten galt ${ }^{18}$, lag es für Poincaré nahe, die Beratungen abzuwarten, bevor er in die Kammerdebatte eintrat. Die Young-Kommission aber legte ihre Vorschläge, in denen zum erstenmal eine faktische Korrelation zwischen deutschen Reparationen und interalliierten Schulden vorgesehen war, am 7. Juni 1929 vor. ${ }^{19}$

Einfluß auf die zögernde Haltung Poincarés, sich dem Thema entschlossen zu nähern, hatten daneben die weitverbreiteten Ratifikationsvorbehalte in der öffentlichen Meinung, die bis ins Parlament hinein ausgestrahlt hatten. War man dort, gestützt auch auf beruhigende Versicherungen der eigenen Staatsführung, seit

14 Artaud, Dettes interalliées, S. 908 f.; Dies., Hintergründe, S. 244-246 (Seiten im Druck vertauscht).

15 Im Rückblick motivierte Poincaré selbst seine Taktik mit der Hoffnung, diese Verknüpfung herstellen zu können; vgl. seine Parlamentsrede in der Ratifizierungsdebatte: JO, Chambre des Députés, Débats parlementaires, 1ère Séance du 12 juillet 1929, S. 2494.

16 Die komplexen diplomatischen Vorgänge können hier natürlich nicht annähernd wiedergegeben werden. Vgl. deshalb: Artaud, Dettes interalliées, S. 66-694; Dies., Hintergründe; Weill-Raynal, Réparations III, S. 365-383.

17 Ebenda, S. 390-392.

18 Ebenda, S. 419f. und 438-461.

19 Ebenda, S. 463-467; dort auch eine Analyse der enthaltenen Ungleichbehandlung des deutschen und der alliierten Schuldner. 
Kriegsende weithin von einem Schuldennachlaß durch die USA ausgegangen, so mußte man nun zur Kenntnis nehmen, daß jener nicht gewährt wurde, während die als Kompensation erhofften deutschen Reparationszahlungen im Young-Plan eine neuerliche Reduzierung erfuhren. ${ }^{20} \mathrm{Da}$ das Thema sensibelste Bereiche der kollektiven Erinnerung an die Leiden und Opfer des Weltkrieges berührte, neigte man in der Öffentlichkeit zu einer irrationalen und schließlich auch ungerechten Betrachtungsweise. Angesichts der amerikanischen Unnachgiebigkeit kursierte das Wort von den Soldaten, „die die Mäntel selbst zu bezahlen haben, in denen sie sich töten ließen". In empörten Stellungnahmen geriet das Bild Frankreichs als Opfer des exzessiven Gewinnstrebens der amerikanischen Kriegsindustrie zu einem regelmäßig zitierten Topos. ${ }^{21}$ Erklärbar ist die Heftigkeit der Attacken nur vor dem Hintergrund eines seit Mitte des 19. Jahrhunderts in der französischen Gesellschaft virulenten, kulturkritisch bestimmten Antiamerikanismus, dem das Schuldenproblem noch vor Ausbruch der Weltwirtschaftskrise ein weiteres konstituierendes Element hinzufügte.22

Unbeschadet der insgesamt dilatorischen Politik Frankreichs im Hinblick auf eine verbindliche Regelung war es zwischen 1927 und Sommer 1929 allerdings zu einer Reihe von provisorischen Vereinbarungen mit Großbritannien und den USA gekommen, in denen sich die französische Regierung zur Leistung von Abschlags- und Zinszahlungen noch vor Inkrafttreten der eigentlichen Verträge verpflichtet hatte. Diese hatten allmählich den Charakter eines „fait accompli“ anzunehmen begonnen und wurden durch den amerikanischen Kongreß am 19. Juni 1929 mit der Zusicherung honoriert, als neues Zahlungsziel für den „kommerziellen" Teil der Schuld den 1. Mai 1930 einzuräumen, falls die französische Kammer die "Accords Mellon-Bérenger" vor dem ursprünglichen Fälligkeitstermin des 1. August 1929 ratifizieren würde. ${ }^{23}$ Bis Frühsommer 1929 hatte man sich im französischen Finanzministerium nach sorgfältiger Prüfung der Alternativen - etwa einer Zahlung der kommerziellen Schuld mit anschließenden Neuverhandlungen - zudem davon überzeugt, daß auch vom finanzpolitischen Standpunkt die Ratifikation die günstigste Lösung darstellte; Poincaré hatte sich jener Position angeschlossen. ${ }^{24}$ Es ging nun darum, Parlament und Öffentlichkeit diese Einsicht nahezubringen.

Unter Zeitdruck und belastet durch die Hypothek eines mißglückten letzten Aufschubsversuchs leitete die Regierung Poincaré am 9. Juli 1928 die parlamentarische Beschäftigung mit dem Washingtoner Schuldenabkommen durch die Vorlage eines Ratifizierungsantrags ein. ${ }^{25}$ Bei Beginn der neuen Sitzungsperiode geriet

20 Bonnefous, Histoire IV, S. 348-350.

21 Vgl. etwa die Kampagne des politischen Publizisten Chéradame in seiner Zeitschrift Le Français Réaliste im Herbst 1928 und seine 1931 veröffentlichte Publikation „Sam, à votre tour, payez“ (Artaud, Dettes interalliés, S. 909).

22 Zur Geschichte antiamerikanischer Tendenzen in Frankreich im Überblick: Michel Winock, Nationalisme, antisémitisme et fascisme en France, Paris 1990, S. 50-76; zu deren Wurzeln im 19. Jahrhundert: René Rémond, Les Etats-Unis devant l'opinion française 1815-1852, Paris 1962.

23 Ebenda, S. 396-399.

24 Artaud, Dettes interalliées, S. 909-912.

25 Die Parlamentsdebatte zeichnet nach: Bonnefous, Histoire IV, S. 348-360; zum Antrag FranklinBouillons, Poincaré zu einem letzten Vorstoß bei der amerikanischen Regierung zu bewegen: S. $350 f$. 
das Thema allmählich in den Blickpunkt der Fraktionen, bevor ab Frühsommer 1929 die parlamentarischen Beratungen einsetzten. Nachdem weder die Finanzkommission noch der Auswärtige Ausschuß der Kammer bereit waren, sich den Regierungsstandpunkt zu eigen zu machen und die Ratifizierung ohne Auflagen zu empfehlen, entschloß sich Poincaré, das Schicksal seines Ministeriums über die Vertrauensfrage an die erfolgreiche Ratifikation der Washingtoner Verträge zu binden. ${ }^{26}$ Die im Vorfeld bereits angekündigte Opposition der sozialistischen Linken und die ablehnenden Stellungnahmen führender Politiker der Mehrheit wie Louis Marin, Louis Dubois, Edouard Soulier und François de Wendel verhießen keinen leichten Kampf. ${ }^{27}$

So entwickelte ein bereits von Krankheit gezeichneter Ministerpräsident in einer über drei Sitzungen vom 11. bis zum 16. Juli 1929 sich erstreckenden, faktengesättigten Parlamentsrede den Regierungsstandpunkt. Im Mittelpunkt stand ein schlagendes Argument: Es könne im Grunde gar nicht die Rede davon sein, sich in der Frage der eingegangenen Schuld zur Nichtanerkennung befugt zu glauben. Letztlich nämlich unterliege lediglich der im Washingtoner Abkommen festgesetzte Zahlungsmodus einschließlich des implizit enthaltenen Schuldennachlasses der Ratifikation durch die Kammer, nicht jedoch die bereits seit langem erfolgte Anerkennung der Schuld selbst. Eine Nichtratifikation könne überdies die im Young-Plan erreichte faktische Abgleichung deutscher Zahlungen an Frankreich und französischer Verpflichtungen gegenüber den USA gefährden. ${ }^{28}$

In der nachfolgenden Diskussion von Änderungsanträgen zum Regierungsentwurf zeigten sich Regierungsmehrheit wie Opposition angesichts der Brisanz der Materie gleichermaßen gespalten. Die wichtigste Mehrheitsgruppierung, die rechtsstehende "Union républicaine démocratique“, zugleich parlamentarisches Sammelbecken der „Fédération Républicaine“ seit der Legislaturperiode 1924$1928{ }^{29}$, hatte darauf verzichtet, einen offiziellen Redner zu bestellen. Louis Marin sprach ebenso wie der ehemalige Vorsitzende der alliierten Reparationskommission, Louis Dubois, nur in eigenem Namen. ${ }^{30}$ Im Gegensatz zum Regierungsentwurf lehnten sie die Ratifizierung ab und forderten die Wiederaufnahme der interalliierten Verhandlungen, ein Standpunkt, dem sich der sozialistische Abgeordnete Vincent Auriol anschloß. Das schlagendste und schlechterdings nicht von der Hand zu weisende "technische“ Argument der Ratifizierungsgegner bezog sich auf die im Young-Plan tatsächlich nur in Form von Transfermodi, doch nicht juristisch eindeutig festgeschriebene Verknüpfung deutscher Reparationszahlungen und französischer Verbindlichkeiten gegenüber den USA. Edouard Herriot als Sprecher des in sich uneinigen Parti Radical monierte ebenso wie der Sozialist Vincent Auriol die Ungleichbehandlung deutscher und interalliierter Schulden bei Moratoriumsbedingungen und Transferkonditionen: Tatsächlich war für den Fall

26 Bonnefous, Histoire IV, S. 352.

27 Jeanneney, de Wendel, S. 420; Artaud, Dettes interalliées, S. $913 \mathrm{f}$.

28 Vgl. die Debattenberichte vom 11., 12. und 16. Juli 1929; hier: JO, Chambre des Députés, Séance du 11 juillet 1929, S. 2463 und Ebenda, Séance du 16 juillet 1929, S. 2545 f.

29 Vgl. Jean Carrère/Georges Bourgin, Manuel des partis politiques en France, Paris 21928, S. 77; Irvine, Conservatism, S. 51.

30 Bonnefous, Histoire IV, S. 353 spricht irrtümlicherweise von einer offiziellen Bestellung Dubois' als Sprecher der „Union Républicaine Démocratique“. 
einer Verminderung alliierter Schuldenzahlungen die Reduzierung auch der deutschen Annuitäten vorgesehen, umgekehrt jedoch kein alliierter Schuldennachlaß bei deutschen Zahlungsproblemen. ${ }^{31}$

Die offizielle Stellungnahme der sozialistischen Fraktion, enthalten in einem Änderungsantrag Léon Blums, zielte als einzige über rein technische Fragestellungen hinaus auf eine umfassendere Regelung. Darin sollten der Truppenabzug aus dem Rheinland, die Annahme des Young-Plans durch Deutschland und die somit wiederum gewährleistete französische Zahlungsfähigkeit gegenüber den alliierten Gläubigern eine enge Verbindung eingehen. Ohne eine solch grundlegende Verankerung der wichtigsten aus dem Krieg resultierenden Probleme in einer allgemeinen Friedenspolitik war man nicht zur Ratifikation bereit. ${ }^{32}$

Am 18. Juli entwickelte Paul Reynaud als Sprecher der „Action démocratique et sociale", einer 31 Mitglieder umfassenden Fraktion der rechten Mitte ${ }^{33}$, seine Sehweise der Sachlage, die in den Reihen der Rechten und der Mitte auf breite Zustimmung stieß.34 Ohne die Berechtigung von Vorbehalten gegen die Verträge abzustreiten, wandte er sich energisch gegen solche, die geeignet sein konnten, die Ratifizierung scheitern zu lassen. Im Vordergrund seiner Argumentation stand das Bemühen, die Debatte weitestmöglich aus dem Reparationskontext herauszulösen und von der rein wirtschaftlichen Ebene auf eine „moralische“ zu verlagern. Es gelte, so Reynaud, die Beziehungen zum Kriegspartner USA nicht zu gefährden, Großzügigkeit walten zu lassen, die Isolierung Frankreichs zu vermeiden und nicht dessen Gegnern in den USA und in Großbritannien in die Hände zu spielen, die bei Neuaufnahme der Verhandlungen auf eine Verschärfung der Bedingungen hofften. Nachdem die vorhergegangene Debatte über weite Strecken auf prozedurale Taktiken zur Relativierung der Ratifikation, zur nachträglichen Verknüpfung französischer Zahlungen mit deutschen Reparationsleistungen oder deren völlige Abwendung abgestellt gewesen war, machte sich Reynaud daran, das Kaleidoskop solcher „petites habiletés procédurières“"35, wie er diese Überlegungen nannte, Punkt für Punkt zu entkräften. Er blieb dabei nicht unwidersprochen durch den zuständigen Rapporteur des Auswärtigen Ausschusses, war aber doch immerhin überzeugend genug, um die Fraktion der „Union républicaine et démocratique" in der Mehrheit von ihrer ablehnend gestimmten Führung abzubringen und den entscheidenden Baustein für einen knappen Sieg der Befürworter einzufügen.

Es sei gleichermaßen irrig, so Reynaud, auf Transferprobleme und eine etwa daraus resultierende amerikanische Ablehnung französischer Zahlungen zu hoffen, wie auch nach dem Einfügen juristisch wirksamer Vorbehaltsklauseln in das

31 JO, Chambre des Députés, Séance du 16 juillet 1929, S. 2543 (Herriot); ebenda, 2e Séance du 18 juillet, S. $2635 \mathrm{f}$. (Auriol). Zu den einschlägigen Passagen des Young-Plans: Weill-Raynal, Réparations III, S. 463-465.

32 Ziebura, Léon Blum, S. 394 geht kaum auf die Debatte ein; vgl. Bonnefous, Histoire IV, S. $354 \mathrm{f}$.

33 André Siegfried, Tableau des partis en France, Paris 1930, S. 176. Die Mitglieder der "Alliance“ verteilten sich in der 1928 gewählten Kammer auf nicht weniger als sieben Fraktionen, zu denen neben der erwähnten "Action démocratique et sociale" etwa auch die rechts von ihr stehende "Union républicaine et démocratique" zählte (Wileman, L'Alliance, S. 383).

34 JO, Chambre des Députés, Débats parlementaires, 2e séance du 18 juillet 1929, S. 2643-2649.

35 Ebenda, S. 2647. 
Ratifizierungsgesetz noch die Annahme durch den amerikanischen Senat zu erwarten. Hieran könnten auch juristische Finessen wie die Zweiteilung des Vorbehaltsartikels nichts ändern. Zudem sei es - wie die Erfahrungen mit dem DawesPlan ja in der Tat gezeigt hatten - höchst unwahrscheinlich, daß es tatsächlich zu der im Debattenverlauf immer wieder angesprochenen, befürchteten Kommerzialisierung der französischen Schuld durch die USA kommen werde, da die dem Mellon-Bérenger-Abkommen inhärenten Bedingungen dafür hinreichend ungünstig seien, um potentielle private Anleger abzuschrecken. ${ }^{36}$ Gegen den Versuch vor allem der S.F.I.O, die Ratifizierungsdebatte als Hebel für den Sturz der Regierung Poincaré zu benutzen, aber auch gegen die Ratifizierungsgegner im Lager der Mehrheit stellte Reynaud rhetorisch wirksam das nationale Interesse: „[...] la ratification est une condition préalable à toute politique, quelle qu'elle soit, politique de droite ou politique de gauche, et les routes bifurquent non pas avant la ratification, mais après la ratification." 37

Am 20. Juli 1929 stimmte die Kammer mit der knappen Mehrheit von nur 300 gegen 292 Stimmen dem Ratifikationsgesetz zum Washingtoner Abkommen zu. Kurz vorher war mit ähnlichen Mehrheitsverhältnissen eine juristisch unverbindliche "motion" verabschiedet worden, die einmal mehr den Anspruch auf Dekkung der französischen Zahlungen durch deutsche Leistungen untermauerte. ${ }^{38}$ Vor die Alternative gestellt, entweder die Regierung Poincaré zu desavouieren und danach die eventuelle Rückkehr einer Linkskoalition in Kauf nehmen zu müssen oder aber der Ratifikation zuzustimmen, hatte sich der Kern der Regierungsmehrheit, das Zentrum und die Rechte, gegen die Stimmen der Sozialisten und die fast geschlossene Opposition der Radikalsozialisten überwiegend für Poincaré entschieden. ${ }^{39}$ Noch mehr als dreißig Jahre später zählte Paul Reynaud genüßlich die Namen derer auf, die Poincaré ihre Unterstützung verweigert hatten: Cachin, Blum, Daladier, Herriot, Franklin-Bouillon, de Wendel, Mandel, Marin und Flandin. ${ }^{40}$

Wenn sich im Verlauf der Debatten überzeugend genug herauskristallisiert hatte, daß ein Mittelweg zwischen beiden Optionen sachlich und „ratifikationstechnisch" nicht gangbar war, so kam das Verdienst hieran in hohem Maße Reynaud zu. Neben Poincaré, Außenminister Briand und Finanzminister Chéron war er der wichtigste Redner gewesen, der der schweigenden Gruppe der Befürworter im Parlament Stimme verliehen hatte. ${ }^{41} \mathrm{Daß}$ dies in gewohnter Gedankenschärfe und rhetorischer Brillanz geschehen war, hatte seine Wirkung sicherlich gefördert, ebenso wie das erworbene Gewicht, das nicht zuletzt aus der Nominierung zum Debattenredner sprach.

36 Ebenda, S. 2646.

37 Ebenda, S. 2648.

38 Abgedruckt in: JO, Chambre des Députés, Séance du 20 juillet 1929, S. 2739. Zur Auswertung des Abstimmungsergebnisses: „La ratification des accords“, in: Le Temps, 22. 7. 1929. Vgl. auch Artaud, Dettes interalliées, S. 917.

$39 \mathrm{Vgl}$. hierzu die Analyse des Entscheidungsprozesses in der Fédération Républicaine bei Jeanneney, de Wendel, S. 432-435.

40 Reynaud, Mémoires I, S. 272.

41 Neben den Genannten hatten unter anderem die Abgeordneten Champetier de Ribes (Démocrates Populaires) und Guernut (Indépendants de Gauche) im Namen ihrer Gruppen, Engerand (Union Républicaine Démocratique) in eigenem Namen für die Ratifizierung gesprochen. 
Nicht übersehen werden darf freilich auch, daß er - entgegen dem Eindruck, den er im nachhinein zu erwecken versuchte - keineswegs alleine einer Phalanx von Gegnern gegenüberstand. ${ }^{2}$ Vielmehr konnte er sich auf eine Meinungsbewegung stützen, die ihm noch vor der Ratifizierungsdebatte wertvolle Bundesgenossen verschafft hatte. So war es beim bedeutendsten Mehrheitsbeschaffer „Fédération Républicaine" buchstäblich am Vorabend der ersten Debattensitzung vom 11. Juli zu einer „Palastrevolution“ gekommen, in deren weiterem Verlauf sich die Parlamentsfraktion der Partei dem Einfluß ihrer bekanntermaßen ablehnend gestimmten Führung um Louis Marin entzogen hatte. Nicht genug, daß man die parlamentarische Redezeit Marins, Dubois' und de Wendels durch den Verzicht auf eine offizielle Rednerbenennung von einer Stunde auf die für private Interventionen vorgesehenen fünfzehn Minuten beschnitten hatte. In der vorentscheidenden Abstimmung über den Antrag Dubois verweigerten am 16. Juli 80 von 100 Abgeordneten der "Union Républicaine Démocratique“ ihrem Parteikollegen die Stimme. In das Ergebnis des Schlußvotums vom 20. Juli schließlich gingen allein 84 befürwortende Stimmen der URD ein. ${ }^{43}$

Auch scheint im Milieu des politiknahen Großhandels und der Industrie, wo der entschiedene Ratifikationsgegner de Wendel weitgehend alleine stand, die gegenteilige Tendenz deutlich die Oberhand gewonnen zu haben, ebenso wie bei den Frontkämpfervereinigungen, die noch im Juni in Großdemonstrationen ihrer Indignation Luft gemacht hatten. ${ }^{44} \mathrm{Als}$ wertvolles Faustpfand auf seiten der Regierung und des eigenen Standpunkts konnte Reynaud zudem die Unterstützung der bedeutendsten Zeitungen liberaler und konservativer Prägung verbuchen, deren Meinungsbildung Poincaré Mitte Juni durch finanzielle Zuwendungen nachgeholfen hatte. ${ }^{45}$

Paul Reynaud hatte bereits am 7. Juni in einem Interview für eines der geförderten Blätter, die konservative Tageszeitung Paris-Midi, seine Argumente konzis zusammengefaßt und einen geschickten Appell an die Parlamentsmehrheit gerichtet. Nachdem die Opposition aus den Reihen der URD nicht ohne Wirkung auf den Reparationsentwurf des Expertenkomitees geblieben sei, so Reynaud, sei es nun an der Zeit, sich in die Realitäten zu fügen: „Après ce nouveau service rendu au pays, la majorité nationale peut se rendre à l'évidence des faits et laisser à l'opposition antigouvernementale la lourde responsabilité d'une mauvaise manoeuvre, laquelle échouera d'ailleurs sûrement.“"46

42 Reynaud, Mémoires I, S. 258-273. Bonnefous, Histoire IV, S. 355-357, der sich vorwiegend auf die Betrachtung der Parlamentsdebatten stützt, hebt ebenfalls die prädominante Rolle Reynauds hervor.

43 Jeanneney, de Wendel, S. 420-423.

44 Ebenda, S. 423 f.; Bonnefous, Histoire IV, S. 350.

45 Laut einem Bericht der amerikanischen Botschaft zählten dazu Le Matin, Le Journal, Le Petit Parisien, Le Petit Journal, L'Echo de Paris, L'Action Française, Le Temps, Le Journal des Débats, L'Intransigeant, La Liberté, Le Figaro und Paris-Midi (zitiert bei Artaud, Dettes interalliées, S. 96, Anm. 105). Zumindest im Falle der militant antiratifikatorisch auftretenden L'Action Française scheint das Unterfangen allerdings vergeblich gewesen zu sein.

46 "Avant la bataille des dettes... Conversation avec le „petit caporal“ de la majorité. M. Paul Reynaud nous dit son plan politique de ratification", in: Paris-Midi, 7.6. 1929; wiederabgedruckt in: La République Démocratique, 16. 6. 1929. 
Daß man diese goldene Brücke genutzt und sich in der Mehrheit zur Ratifizierung bequemt hatte, verschaffte Reynaud einen der größten Erfolge seiner bisherigen politischen Laufbahn. Der geglückte Coup machte ihn zu einem der neuen Meinungsführer im regierungstragenden Lager von der rechten Mitte bis zur republikanischen Rechten, und zwar gegen einen Louis Marin, der sich mit seinen extrem nationalistischen Ansichten einmal mehr selbst ins Abseits gestellt hatte. Wieder einmal auch hatte Reynaud damit einem Gegner von Gewicht den Fehdehandschuh hingeworfen.

1871 im lothringischen Faulx geboren, gehörte der Jurist und Anthropologe Louis Marin zum parlamentarischen „Urgestein“ der Dritten Republik. Seit 1905 vertrat er das 2. Arrondissement von Nancy in der Kammer und nahm dieses Mandat in der Folge über nicht weniger als 46 Jahre hinweg wahr. Er profilierte sich als hart arbeitender, ungewöhnlich wohlinformierter Abgeordneter, dessen Autorität ihn in seinem politischen Leben an die Spitze einer großen Zahl von Parlamentskommissionen getragen hatte. Wenn ihn die sechs Ministerämter seiner Laufbahn indes nie an wirkliche Schlüsselpositionen führten, so lag der Grund dafür hauptsächlich in seiner hypertrophen nationalistischen Grundhaltung. Sie machte ihn in den Augen des liberalen Zentrums zu einer "bête noire“ des politischen Lebens, die man im Rahmen bestimmter koalitionspolitischer Notwendigkeiten in der gemeinsamen Regierungsmannschaft eher ertrug als wirklich schätzte. Seine bereits sprichwörtliche Germanophobie hatte ihn 1919 als einen von zwei nichtsozialistischen Abgeordneten gegen den Versailler Vertrag votieren lassen, da er ihn als zu gemäßigt einstufte. Sie machte ihn in der Folge zum Verfechter seiner kompromißlosen Ausführung und naturgemäß zum entschiedenen Gegner der Versöhnungsansätze Briands. Mit dem Sieg des Linkskartells war er im Zuge der allgemein auf der Rechten zu beobachtenden organisatorischen und ideellen Umstrukturierung im Juni 1925 an die Spitze der „Fédération Républicaine" gelangt, die er in kurzer Zeit durch seine politische Persönlichkeit prägte. Sein autokratischer Führungsstil und sein oft selbstgerechtes Beharren auf einmal eingenommenen Positionen hatten ihm in dieser Funktion nicht nur Freunde geschaffen, insbesondere unter der jüngeren Generation von Parteimitgliedern wie Henri de Kerillis. ${ }^{47}$ Im Juli 1926 war er von Poincaré als Minister für die Soldatenpensionen in seine Regierung der "Union nationale“ aufgenommen worden, um durch dieses Zugeständnis die Unterstützung der „Fédération Républicaine“ zu gewinnen. Im Kalkül des Ministerpräsidenten, dessen Abneigung gegen den nationalistischen Flügel seiner Mehrheit notorisch war, hatte Marin vor allem die Rolle eines Moderators zu erfüllen, der allzu forsche deutschlandpolitische Vorstöße der Linken und Briands einzudämmen und Poincaré damit von einem persönlichen Eingreifen weitgehend zu dispensieren hatte. ${ }^{48}$ Nach dem Austreten der radikalsozialistischen Minister aus dem Kabinett im November 1928 hatte Poincaré dann auch Marin geopfert, um damit die Loyalität der mehr und mehr in die

47 Nachdem eine aus den verfügbaren Quellen gearbeitete Gesamtbiographie Marins nach wie vor nicht vorlicgt, sind als neueste Darstellungen heranzuziehen: Irvine, Conservatism, S. 6-11; JeanFrançois Eck, Louis Marin, in: Jean-François Sirinelli (Hg.), Dictionnaire historique de la vie politique française au XXc siècle, Paris 1995, S. 630 f.; Jeanneney, de Wendel, S. 427-431 und passim.

$48 \mathrm{Zu}$ diesem Aspekt des Verhältnisses Poincaré-Marin: Jeanneney, de Wendel, S. $364 \mathrm{f}$. 
Opposition abgleitenden Radicaux zu erhalten. ${ }^{49}$ Der damit verbundene Autoritätsverlust Marins bildete die Voraussetzung für die nachfolgende Insubordinationsbewegung unter dessen innerparteilichen Gegnern, zu denen neben Henri de Kerillis auch de Lasteyrie, de Monicault und d'Audiffret-Pasquier zählten.

Im Zuge der Ratifikationsdebatte hatte die Gruppe um Marin und de Wendel der vermeintlich besseren Option im Sinne des nationalen Interesses den Vorrang vor der Einheit der Partei und vor dem Erhalt der Regierung Poincaré gegeben, die doch ihren Zielen zumindest als "geringstes Übel“ weitgehend entsprach.50 Die Orientierungskrise in der „Fédération“, die anläßlich der Schuldenfrage manifest wurde und während der gesamten Legislaturperiode fortdauern sollte, hatte zur Spaltung der Partei, ja zu ihrer Lähmung geführt und neuen Kräften den Weg bereitet. Paul Reynaud gehörte zu jenen, die davon unmittelbar profitiert hatten um den Preis freilich eines nach dem Wort Daladiers „brudermörderischen Kampfes" ${ }^{51}$ mit Louis Marin. Daß er in dieses Kräftemessen ganz wider Willen eintrat, ist nicht anzunehmen. Sicherlich hatte er der „Fédération“ und ihrem Parteichef nach 1926 die Hand zum dankbar akzeptierten Wahlbündnis gereicht und damit deutlich größere Affinitäten gegenüber dem prinzipientreuen Nationalisten gezeigt als die eigene Parteiführung in der „Alliance Républicaine Démocratique “.52 Dennoch konnte es keinerlei Zweifel unterliegen, daß ihn etwa in der Deutschlandfrage ein unüberbrückbarer Graben von Louis Marin trennte. Vor allem auf diesem Gebiet kam es in den dreißiger und noch in den vierziger Jahren wiederholt zu Kontroversen zwischen beiden Politikern: im Jahr 1938 etwa, als Marin im Unterschied zu Reynaud die Beschwichtigungspolitik Bonnets gegenüber Deutschland unterstützte, und noch im Juni 1948, als beide Politiker in der Kammerdebatte um die Ratifizierung der Londoner Empfehlungen mit gegensätzlichen Vorstellungen aufeinandertrafen. Gravierende Folgen für das politische Schicksal Reynauds hatte die Haltung Marins im Frühjahr 1940: Da der Parteichef der „Fédération“ sich weigerte, in Reynauds am 21. März gebildete Regierung einzutreten, fehlte ihr damit von vorneherein die Unterstützung der parlamentarischen Rechten - mit gravierenden Folgen für ihr weiteres Schicksal. Die Wurzeln aber für ein solcherart auch persönlich problematisches Verhältnis der beiden Politiker lagen im wesentlichen in den Ereignissen des Sommers 1929. Bei Beobachtern ganz entgegengesetzter politischer Provenienz glaubte man zu diesem Zeitpunkt klare Anzeichen dafür auszumachen, daß Louis Marins politischer Einfluß endgültig im Sinken begriffen sei. In der Tat übernahm mit den ausgehenden

49 Ebenda, S. $418 \mathrm{f}$.

50 Ebenda, S. 434 f. und 364 f. (Zitat: S. 365).

51 Zitiert nach Jeanneney, de Wendel, S. 421.

52 Ein Informantenbericht vom Juni 1927 beschreibt die Haltung der Parteiführung der „Alliance“ gegenüber der „Fédération“ so: „C'est avec ces éléments-là [mit den rechten Radikalsozialisten; A.d.V.] que M. Ratier, M. Flandin, reprenant la manoeuvre préconisée par M. Mamelet, voudraient se grouper, et non avec les nationaux de la Fédération Républicaine qu'ils supportent beaucoup plus qu'ils ne les recherchent. A leurs yeux, en effet, la Fédération ne représente pas seulement l'aile droite de l'Union Nationale Républicaine mais le parti qui reprend peu à peu la politique et le programme plus ou moins avoués des cléricaux et des anciens nationalistes." Bericht vom 25.6. 1927 (AN, $\mathrm{F}^{7} 13237$ ).

Zu den positiven Reaktionen in der "Fédération Républicaine“ auf die Avancen vgl. den Polizeibericht „A. 2891. A/s du Congrès de la Fédération Républicaine“, 12.12. 1927 (AN, F¹3962). 
zwanziger Jahren und mit dem Abtreten von Persönlichkeiten wie Poincaré und Briand allmählich eine neue Generation die Schlüsselpositionen in der Politik Frankreichs. Das kursierende Gerücht, wonach Reynaud der verantwortliche Drahtzieher für den Abfall der „groupe Marin“ gewesen sei, mag von der „Action Française" böswillig in Umlauf gebracht worden sein; doch nicht nur dort war man mit gutem Grund davon überzeugt, daß einer der neuen führenden Männer der parlamentarischen Mehrheit nun Paul Reynaud hieß.

In der Pariser Presse nahm man die Machtkämpfe im Lager der "modérés“ mit Neugier und einem Anflug von Ironie zur Kenntnis, die sich im Wort vom „petit caporal de la majorité" widerspiegelte, das man Reynaud zudachte. ${ }^{33}$ Das Zusammentreffen von geringer Körpergröße und herausgehobener Position, von persönlichem Ehrgeiz und Karrierestreben in seiner Person konnte die Anspielung auf den Beinamen Napoléon Bonapartes durchaus nahelegen. Der Vergleich hinkte dort, wo er die handstreichartige „Usurpation“ der Macht als ausschlaggebenden Faktor für den politischen Aufstieg Reynauds suggerieren sollte.

Es bedurfte einer ganzen Reihe von Voraussetzungen und Fähigkeiten, um politisches Gewicht in einem System zu erlangen, das nicht von der Dominanz überregionaler Parteien und ihrer Apparate, sondern von jener des Parlaments geprägt war. Als Instrumente basisverbundener politischer Elitenbildung spielten allenfalls die Linksparteien eine gewisse Rolle. Die „Alliance“ pflegte weder den entsprechenden Anspruch noch verfügte sie über das personelle Reservoir an „militants", um vergleichbare Funktionen zu übernehmen. Eine Parteikarriere von der Pike auf war in dieser Gruppierung nicht möglich, ihre Bedeutung als Instrument zur integralen Förderung politischer Karrieren deshalb begrenzt. Der Werdegang Reynauds bis ins Jahr 1929 illustriert dieses Faktum. Für ihn wurde die Zusammenarbeit mit der "Alliance“ überhaupt erst von Wert und Interesse, als er bereits auf nationaler Ebene politisch arriviert war. Dann aber lag es aus seiner Sicht nahe, sich der bestehenden Strukturen da zu bedienen, wo sie ein Mindestmaß an Tragkraft aufzuweisen schienen: im hauptstädtischen politischen Getriebe, wo er bei den Nachwahlen von 1926 erstmals als Kandidat der „Alliance“ auftrat, und im Rahmen der parlamentarischen Arbeit seit 1920/21. Bis dorthin und weit darüber hinaus aber lassen sich in Reynauds Laufbahn auch andere Faktoren von mindestens gleichrangiger Bedeutung für Vorankommen und politischen Aufstieg ausmachen. In mancher Hinsicht überlagerten sich darin traditionelle Karrieremuster mit Elementen jüngerer Art. ${ }^{54}$

53 Paris-Midi, 7.6. 1929 (Zitat). Vgl. dazu etwa die Lageanalysen: „Le réprouvé“, in: Le Populaire, 20. 7. 1929; L'Action Française, 20. 7. 1929.

54 Die Genese und Struktur parlamentarischer Eliten im Frankreich der Zwischenkriegszeit war, abgesehen von regional orientierten Studien, bislang noch kein Schwerpunktthema der historischen Forschung. Leider von begrenztem Nutzen ist die bislang ungedruckte Studie von Gilles Le Béguec, L'entrée au Palais Bourbon: Les filières privilégiées d'accès à la fonction parlementaire (19191939). Thèse d'Etat, Paris 1989; in komprimierter und thematisch zugespitzter Form erneut: ders, De la république des avocats à la république des énarques, in: Louis Dupeux/Rainer Hudemann/ Franz Knipping (Hg.), Eliten in Deutschland und Frankreich im 19. und 20. Jahrhundert. Strukturen und Beziehungen. Elites en France et en Allemagne aux XIXème et XXème siècles. Structures et relations, Band II, München 1996, S. 79-89.

Systematischer und in Anlehnung an Bourdieus Konzept des „capital culturel“ hat François Grèzes-Rueff versucht, anhand eines ausgewählten Samples von Abgeordneten der III. und IV. Repu- 
Enge verwandtschaftliche und politische Beziehungen ins regionale Honoratiorenmilieu hatten die Grundlage für seinen erfolgreichen Wahlkampf von 1919 gelegt, und solide Verbindungen in Kreise politiknaher Pariser Juristen kamen ihm seit seiner Heirat mit Jeanne Henri-Robert weiterhin zupaß. Ungewöhnlich genug angesichts einer Mehrzahl von Abgeordneten, die in der Regel dem Département treu blieben, das sie einmal entsandt hatte, schaffte Reynaud bis 1928 den Sprung nach Paris. Der Weg aus dem wenig entwickelten, überwiegend agrarisch strukturierten südfranzösischen Wahlkreis ins mittelständisch geprägte Zentrum der Hauptstadt war sicherlich biographisch angelegt, bildete doch der Geburtsort Barcelonnette schon seit langem nicht mehr den Lebensmittelpunkt des ambitionierten Anwalts. Er brachte darüber hinaus das mittlerweile gewonnene politische Profil zum Ausdruck, den Ehrgeiz auch, sich aus der zweiten Reihe einmal erfolgreicher und dann 1924 abgewählter Politiker des rechtsliberalen Spektrums herauszuarbeiten. Anders als vielleicht zu erwarten, erwiesen sich die Eigenheiten des 1927 wiedereingeführten Mehrheitswahlrechts im Arrondissementsrahmen dabei nicht als hinderlich. Im Gegenteil: Reynauds politischer Wahlkampfstil war auf die Profilierung vor Ort, nicht auf den Weg über Parteienlisten ausgerichtet, und das neue Wahlrecht belohnte all jene Kandidaten, die sich eben darauf verstanden. ${ }^{55}$ Daneben profitierte der Politiker davon, daß er sich vor dem Zweiten Weltkrieg stets nur einem relativ begrenzten Wählerkreis zu stellen hatte. Im Jahre 1919 zählte sein Département Basses-Alpes mit etwas über 31000 Wahlberechtigten zu den am schwächsten besetzten Frankreichs; in seinem Pariser Wahlkreis traf Reynaud bei der Wiederwahl von 1928 auf etwa 12000 eingetragene Wähler. Diese Tatsache allein konnte Niederlagen wie jene von 1926 natülich nicht verhindern, erleichterte ihm aber unter den Bedingungen der annähernden bzw. reinen Persönlichkeitswahl jeweils den Neuanfang im Wahlkreis. ${ }^{56}$

Über diese punktuell wirksamen oder sehr persönlichen Elemente eines politischen Werdegangs hinaus zeichnen sich zwei allgemeinere Qualifikationsstränge $\mathrm{ab}$, entlang derer sich die Karriere Paul Reynauds entwickelte. Bis Mitte der zwanziger Jahre hatte er einen makellosen, republikanischen "cursus honorum" zurückgelegt, wichtig für Ansehen und Wahlchancen. Das Amt des „Conseiller

blik mehrere Parlamentarierkategorien von jeweils gemeinsamer politischer „Kultur" zu fassen: François Grèzes-Rueff, La culture des députés français (1910-1958). Essai de typologie, Toulouse 1994. Aus den ab den fünfziger Jahren geführten, theoretisch gehaltenen Elitendebatten zwischen Soziologen und Politologen gingen auch einige historisch orientierte Darstellungen hervor, darunter: Pierre Birnbaum, Les sommets de l'Etat. Essai sur l'élite du pouvoir en France, Paris 1977, S. 30-47. Dazu zählen auch die Arbeiten von Mattei Dogan, auf deren statistische Erhebungen die Forschung bezeichnenderweise bis heute angewiesen ist, etwa: Mattei Dogan, Les filières de la carrière politique en France, in: Revue française de Sociologie 8 (1967), S. 468-492. Ein Standardwerk, doch beschränkt auf die Entstehungsphase der Republik: Christophe Charle, Les Elites de la République (1880-1900), Paris 1987.

Bereits ein Klassiker, verfaßt von einem Zeitgenossen und voller interessanter Beobachtungen: Robert de Jouvenel, La République des camarades, Paris 1914. Polemischer und vom Standpunkt konservativer Parlamentarismuskritik aus: André Tardieu, La profession parlementaire, Paris 1937.

55 Zur Analyse des Wahlrechts und zu den Diskrepanzen zwischen Wählervotum und Kammerzusammensetzung, die daraus resultierten: Lachapelle, Elections législatives, 22-29 avril 1928, S. V-XV.

56 Vgl. ebenda, S. 246 f.; ders., Elections législatives du 16 Novembre 1919, S. 46; zu den Wahlkreisdifferenzen im Jahr 1928 auch: Mayeur, Troisième République, S. $286 \mathrm{f}$. 
Général" stand am Anfang. Mehr und mehr war es seit Beginn des Jahrhunderts üblich geworden, den Eintritt in die nationale Politik über kommunale oder regionale Funktionen anzubahnen ${ }^{57}$, und Reynaud hatte die damit verbundene Chance durch ambitionierte Arbeit genutzt. Dazu kamen in der Folge der Dienst an der Republik im Weltkrieg und in verschiedenen diplomatischen Missionen, das publizistisch-kämpferische Eintreten für den republikanischen Wertekanon und gegen die „Action Française“, die öffentlichkeitswirksamen Auftritte mit den kommunistischen Gegenkandidaten im Wahlkampf von 1926: Heterogene Elemente dieser Art addierten sich zu einem Bild verläßlicher republiktragender Gesinnung und ihrer erfolgreichen Umsetzung in den Institutionen und im politischen Alltag. Der theoretische und praktische Rekurs auf ein wenig präzise gehaltenes republikanisches Referenzsystem hatte also über sein politisch mobilisierendes Potential hinaus karrieresteuernde Wirkung. Für den Aufstieg im liberalen Lager bildete er eine notwendige, wenngleich alleine noch nicht hinreichende Bedingung. 58

Spezifischer noch und zugleich präziser faßbar in der Bedeutung für parlamentarische Karrierewege war die traditionell enge personelle und funktionale Verflechtung juristischer Berufe und insbesondere des Pariser Anwaltsmilieus mit der nationalen Politik. Allein die numerische Präsenz von Angehörigen des Rechtsmetiers in den Abgeordnetenkammern der Dritten und Vierten Republik war überdurchschnittlich hoch: Knapp ein Drittel der zwischen 1910 und 1958 aktiven Parlamentarier verfügte zumindest über eine „Licence en droit“. Über die Legislaturperioden hinweg ging ihre Zahl zwar tendenziell zugunsten anderer sozioprofessioneller Gruppen zurück, etwa der Mediziner, der Gymnasial- und Volksschullehrer und der kleinen Angestellten, nach 1945 auch der Arbeiter. Doch verkörperten sie weiterhin zusammen mit den Angehörigen freier Berufe insgesamt und dem mittleren wie kleinen Beamtentum die schrittweise erfolgte Ablösung älterer parlamentarischer Eliten; diese hatte seit Beginn der Dritten Republik zu Lasten von Besitzbürgertum und hohen Staatsbeamten Raum gegriffen. ${ }^{99}$

Die Gründe für die fortbestehende Dominanz lagen zum großen Teil in allgemeineren, strukturellen Ähnlichkeiten zwischen den zeitgenössischen juristischen Ausbildungs- bzw. Tätigkeitsfeldern und der parlamentarischen Arbeit. Das Bild vom Abgeordneten als Anwalt der Wählerinteressen wirkte ebenso karrierefördernd wie die ganz praktische Routine rechtskundiger Volksvertreter im Umgang mit komplizierten gesetzestechnischen Materien. Die ohnehin ausgeprägte rhetorische Kompetenz juristischer Berufe kam im Rahmen der parlamentarischen Kultur Frankreichs ganz besonders zum Tragen. Keineswegs zufällig hatte ein juristischer Rhetorikwettbewerb - die "Conférence du stage" - das Ansehen eines Ausleseinstruments für Anwärter auf Spitzenpositionen in Staat und Politik ge-

57 Zwischen 1900 und 1940 verfügten mehr als zwei Drittel der gewählten Abgeordneten über einen Sitz in einem Stadtrat oder Generalrat; vgl. Birnbaum, Sommets de l'Etat, S. 40.

5* Zu den Elementen cines republikanischen Karrieremusters und zur Bedeutung der Weltkriegsteilnahme bei den Wahlen von 1919: Roussellier, Parlement de l'éloquence, S. 43-48.

59 Birnbaum, Sommets de l'Etat, S. 30 ff.; Grèzes-Rueff, Culture des députés français, S. 101-125; Dogan, Les filières de la carrière politique. 
wonnen.60 Wer hier wie Paul Reynaud reüssierte, hatte sich in den Augen des „Palais" und der interessierten Öffentlichkeit für höhere Aufgaben qualifiziert: Er konnte der Aufmerksamkeit einflußreicher Gönner wie der weiteren Förderung sicher sein. "Jeune homme, lorsque l'on sait parler à la Conférence, on sait parler partout.“"61 - Die herablassende Bemerkung des Präsidenten der Pariser Anwaltsvereinigung gegenüber dem frischvermählten Paul Reynaud enthielt etwas von dieser Hochschätzung des juristisch geschulten Redners. Im politischen Leben Frankreichs war sie durchaus repräsentativ noch zwischen den Weltkriegen und darüber hinaus.

Freilich galt das Karriereschema, das ein Zeitgenosse Reynauds aus der politischen Erfahrung des frühen 20. Jahrhunderts sehr pointiert formuliert hatte, nicht mehr uneingeschränkt: „Il fallait être bachelier pour être licencié en droit, licencié en droit pour être avocat, avocat pour être député, député pour être notable dans les commissions, rapporteur de commission pour être ministre, ministre pour l'avoir été, rester important, avoir la publicité qui assure le retour intermittent au pouvoir."62 Ein neuer Typus von Abgeordneten hatte begonnen, sich zumindest in den parlamentarischen Führungsrängen bemerkbar zu machen, und Paul Reynaud gehörte dazu. Wie die Mehrzahl jener juristisch versierten Parlamentarier, die im Laufe ihrer Karriere in wichtigere politische Positionen gelangten, hatte er seine juristische Ausbildung durch weitere Qualifikationen ergänzt, die in der politischen Arbeit zur Anwendung gebracht werden konnten. Das Studium der Wirtschaftswissenschaften und die Promotion über ein handelsrechtliches Thema hatten ihm ein Maß an ökonomischem Sachverstand gesichert, das in der Kammer der Abgeordneten bis in die dreißiger Jahre noch selten anzutreffen war. Es verschaffte ihm gerade in den zahlenmäßig zunehmenden finanz- und reparationspolitischen Debatten wachsende Autorität. ${ }^{63}$

In dieser Hinsicht stand Reynaud für eine neue Elitengeneration in der Gruppe der „députés juristes“: einer Generation, in der gegenüber Politikern wie Poincaré oder Briand der Erwerb technischer Expertenkenntnisse allmählich die klassischhumanistisch und stark rhetorisch orientierte Ausbildung ergänzte und verdrängte. Gleichwohl blieb der Typus des politisch aktiven Spezialisten der Verwaltungs- und Staatswissenschaften, wie ihn einige Jahrzehnte später Michel Debré verkörperte, in der Kammer vorerst noch die Ausnahme. Auch hier ist Reynauds Beispiel charakteristisch. Zum kleinen Kreis führender Parlamentarier zählte Reynaud in erster Linie deshalb, weil er auf traditionell hochbewerteten Kompetenzfeldern brillierte. Legt man eine Typologie zugrunde, die die intellektuelle Wertschätzung eines Parlamentariers in der Kammer anhand des jeweiligen Anteils an einer in sich vielgestaltigen „Abgeordnetenkultur" zu fassen versucht, dann reüssierte Reynaud aufgrund von Schlüsselqualifikationen in den drei seit dem 19. Jahrhundert essentiellen Bereichen: dem juristischen, dem literarisch-

60 Gilles Le Béguec, L'aristocratie du barreau, vivier pour la République. Les secrétaires de la Conférence du stage, in: Vingtième Siècle 30 (1991), S. 22-31.

61 Reynaud, Mémoires I, S. 94.

62 Anatole de Monzie, L'Entrée au forum: vingt ans avant, Paris 1920, S. 239.

63 Grèzes-Rueff, Culture des députés français, S. 108f; Julian Jackson, The politics of depression in France 1932-1936, Cambridge 1985, S. 12 f. 
historischen und dem rhetorischen. ${ }^{64}$ Reynaud war ein Mann des Übergangs, der sich seit dem Beginn seiner politischen Laufbahn parlamentarismuskritischen Zeitströmungen und damit der an das politische Personal insgesamt gerichteten Kompetenzfrage zu stellen hatte. Er antwortete darauf mit einer Reihe von Reformansätzen, zu denen die Revision der Kammergeschäftsordnung oder, wie noch zu zeigen sein wird, Überlegungen zur Neubalance des Verhältnisses von Regierung und Parlament gehörten. Den forcierten Rückgriff auf das ausgewiesene Expertentum als Lösungsparadigma für neuartige Problemstellungen der Zwischenkriegszeit lehnte er nicht ab; während seiner Amtszeit als Finanzminister in der Regierung Daladier sammelte er einen „brain-trust“ junger Wirtschaftsfachleute um sich, durch den manch alte Kontakte der Vorjahre institutionalisiert wurden. ${ }^{65}$ Ein Technokrat in politischen Führungsrängen war er jedoch selbst nicht und er wollte es auch nicht sein. Sein Idealbild war umfassender und trug manche Züge der Profile führender Liberaler des 19. Jahrhunderts, darunter die persönlich-berufliche Autonomie oder die klassisch-rhetorisch orientierte Ausbildung.

Bei allem Neuerungsanspruch absolvierte Paul Reynaud eine parlamentarische Karriere im traditionellen Stil. Ein gewisses Maß an Konformismus in der Wahrnehmung von Aufstiegschancen kam dabei sicher zum Tragen. Nicht zu vernachlässigen ist aber auch, daß sein politisches Selbstverständnis sich mit der im deliberativen System geforderten Politikerrolle weitgehend deckte. Reynauds Verhältnis zur parlamentarischen Rhetorik mag hierfür als Illustration dienen.66

Reynaud pflegte zeit seines Lebens in mündlichen Äußerungen und in Publikationen den gehobenen Stil und verstand sich gleichzeitig auch auf diesem Feld als Neuerer, als Vertreter einer moderneren Art von Rhetorik: spartanischer, direkter, ohne den üblichen Zierat an Zitaten aus den „Klassikern“ und - wiederum - orientiert am Vorbild Waldeck-Rousseaus. Diese Selbsteinschätzung allerdings traf allenfalls im Verhältnis zu den hypertrophen Kunstformen juristischer Redekunst zu, wie Reynaud sie noch in den Jahren vor dem Ersten Weltkrieg erlebt hatte und im Rückblick beschrieb.67 Auch für ihn gehörte die geschliffene Redewendung, gehörten gelehrte Anspielungen auf die antike Mythologie oder die französische Geschichte und Literatur zum rhetorischen Standardrepertoire, das er regelmäßig entfaltete, und dies nicht nur, um die persönlichen Defizite an körperlicher Imposanz und Tragkraft der Stimme auszugleichen. ${ }^{68}$ Reynaud war williger Erbe und

64 Vgl. dazu den Ansatz von Grèzes-Rueff und besonders die entsprechende Kategorisierung der Abgeordneten der III. und IV. Republik: Grèzes-Rueff, Culture des députés français, S. 163 f., $170-172$.

65 Vgl. Gérard Brun, Technocrates et technocratie en France, 1918-1945, Paris 1985, S. 167-169 sowie Kapitel X.2. dieser Arbeit.

66 Die Beschäftigung der Forschung mit der französischen politischen Rhetorik des 20. Jahrhunderts erfolgte bislang stark unter dokumentarischen, quantitativen oder textlinguistischen Gesichtspunkten: vgl. etwa Sylvianne Rémi-Giraud (Hg.), Les mots de la nation, Lyon 1996; Jacques Guilhaumou, La rhétorique du discours, objet d'histoire (XVIII ${ }^{-}-X^{c}$ siècles), Lille 1981 . François Grèzes-Rueff bereitet zur Zeit eine Thèse über die Rolle des historischen Arguments im parlamentarischen Diskurs vor.

67 Vgl. dazu das Memoirenkapitel, das Reynaud seinen Anfängen im Pariser Anwaltsmilieu widmete: Reynaud, Mémoires I, S. 86-94.

68 Ebenda, S. 92. 
Träger einer französischen Rhetoriktradition, die in ihrer parlamentarischen Anwendung das logische Gegenstück zum Ideal des deliberativ ausgerichteten Parlamentarismus bildete. Der prinzipielle Vorrang des Parlaments im politischen Kräftespiel, dessen Bedeutung als Ort der geregelten diskursiven Auseinandersetzung möglichst unabhängiger Persönlichkeiten, die Identifikation von Repräsentation und Diskussion, das Überzeugenwollen durch die Kraft der Vernunft und die Macht des Worts - das blieben Grundannahmen, an denen er bei allen Reformvorstößen im einzelnen festhielt, Grundannahmen auch, die bereits im Bereich einer tieferliegenden politischen Mentalität ihren Sitz hatten. Wer so dachte, für den war selbstverständlich, daß eine herausgehobene Karriere in der Volksvertretung in ganz besonderem Maße an die rednerische Bravourleistung in der Debatte geknüpft war. Parlamentarischer Einfluß manifestierte sich zwangsläufig unter anderem in der Fähigkeit eines Redners, die fluiden Fraktionsstrukturen zu nutzen und hinreichend Stimmen aus benachbarten Lagern für das Zustandekommen einer sachbezogenen Ad-hoc-Mehrheit zu mobilisieren - selbstverständlich nach vorbereitender Überzeugungsarbeit hinter den Kulissen. Eben in dieser Kunst hatte sich Reynaud im Juli 1929 erfolgreich bewiesen.

Die gewonnene Position trug ihn in fast logischer Zwangsläufigkeit ins erste Ministeramt, als mit André Tardieu wieder ein Regierungschef aus den Reihen der gemäßigten Rechten antrat. Leicht retardierende Wirkung auf dem Wege dahin hatte allerdings die öffentliche Diskussion, die im Herbst 1929 in bezug auf Reynauds wenige Monate zuvor wiederaufgenommenes, aktives deutschlandpolitisches Engagement einsetzte. In ihrer Heftigkeit in erster Linie ein Beleg dafür, daß das politische Tun und Lassen des Politikers mittlerweile im Blickpunkt einer weiteren Öffentlichkeit stand, bezeichnete die Debatte seine Stellung im politischen Kräftefeld und wurde ihm gleichzeitig zum Ausgangspunkt für ihre weitere deutschlandpolitische Ausdifferenzierung.

\section{2. „Néo-nationalisme“ als Krisenlösungskonzept: Pläne und Gespräche für einen Interessenausgleich mit der deutschen Rechten 1929/30}

\section{a) Reynauds Deutschlandbesuch im Frübjabr 1929 und die Reaktionen in Frankreich}

Während einer sechswöchigen Auslandsreise in den USA und in Mexiko erreichte Paul Reynaud Ende September 1929 die Nachricht, daß in Paris eine heftige Pressekampagne gegen ihn im Gange sei. Stein des Anstosses waren die Gespräche, die er im April des gleichen Jahres auf Einladung von Arnold Rechberg in Berlin geführt hatte. Aufgrund von innenpolitisch motivierten Enthüllungen in der deutschen Presse, insbesondere in Stresemanns Nationalliberaler Korrespondenz, im Blatt des Jungdeutschen Ordens und in einer nachfolgenden Presseerklärung deutschnationaler Politiker war publik geworden, daß er Ende April in Unterredungen unter anderem mit einem Vertreter des „Stahlhelm“ und einem Mitar- 
beiter Hugenbergs über Deutschland und Frankreich betreffende außenpolitische Fragen gesprochen hatte. Nach ersten Berichten der Pariser Presse war es dabei zu weitgehenden, allerdings nur in persönlichem Namen erfolgten Vorschlägen Reynauds im Hinblick auf eine Reduzierung der Annuitäten des Dawes-Plans, auf die Rückgabe des polnischen „Korridors“ an Deutschland und ein deutsch-französisches Militärbündnis gekommen. ${ }^{69}$

In einem kontinuierlich sich verschärfenden Crescendo nahmen Blätter der Linken, der linken Mitte und der extremen Rechten diese Enthüllungen vom 23. September an zum Anlaß, den neuen Mehrheitsführer mit scharfer Kritik zu belegen. Einhellig und nur in der Wahl der Argumente abgestuft verurteilten die Parteizeitungen des PCF und der SFIO die Initiative ebenso wie Blätter im Umkreis der radikalsozialistischen Partei oder die antirepublikanische L'Action Française. In den wenigsten ablehnenden Stellungnahmen drang man dabei allerdings zu einer wirklichen Diskussion der außenpolitischen Wünsch- oder Machbarkeit der vermeintlich besprochenen Inhalte vor. Im Mittelpunkt stand vielmehr die Entrüstung über die Tatsache des Kontakts mit der nationalistischen Rechten in Deutschland, die man je nach politischem Standort unter dem Aspekt der klassenoder nationalstaatsbezogenen Gegnerschaft in den Blick nahm. Wo L'Humanité die antisowjetische Wendung der Allianzpläne kritisierte, entwarf Le Populaire unter der Wortführung von Paul Faure das Szenario einer unter dem Vorzeichen der „Internationale du sang" vereinten deutsch-französischen Kriegsindustrie zum Schaden der arbeitenden Klassen beider Länder. ${ }^{70}$ L'Action Française führte die „Machenschaften“ Reynauds mit den „schlimmsten Feinden Frankreichs“ auf dessen Bestreben zurück, eine „führende Rolle zu spielen“ und kritisierte einmal mehr die Schwäche der Regierung, die solche Alleingänge zulasse. ${ }^{71}$ In der radikalsozialistischen und der unabhängigen linken Presse betonte man, wie beispielsweise Léon Blum im Populaire, die Andersartigkeit des eigenen Verständigungsansatzes ${ }^{72}$ und begrüßte Reynaud ironisch im Lager der „Ausgleichswilligen“.73 Vereinzelt nur setzte man sich mit den nicht von der Hand zu weisenden Rückwirkungen der Pläne auf die europäische Staatenordnung und mit den machtpolitischen Reaktionen auf eine deutsch-französische Interessenskoalition auseinander. $^{74}$

69 Vgl. „Une histoire de tractations franco-allemandes“, in: Le Temps, 23. 9. 1929; „Un patriote à l'oeuvre“, in: L'Action Française, 23. 9. 1929; „D'étranges tractations“, in: Le Soir, 23. 9. 1929; „Les tractations qui préparent la guerre“, in: L'Humanité, 23. 9. 1929.

Auch in der deutschen Diplomatie begann man bereits früh auf die „Enthüllungen“ aufmerksam zu werden, Botschaftsrat Rieth nahm die Reaktionen zum Anlaß für mehrere ausführliche Presseberichte (Rieth an AA, 23. 9. 1929 und 25. 9. 1929; PA-AA, R 70562).

70 „Une entente militaire franco-allemande contre les Soviets“, in: L'Humanité, 23. 9. 1929; „Les tractations „patriotiques" de Paul Reynaud“, in: Le Populaire, 24. 9. 1929; „Le drapeau sur la marchandise“, Ebenda, 30.9.1929 (Zitat); „Pacifisme d'affaires“, Ebenda, 11. 10. 1929.

71 „Un patriote à l'oeuvre“, in: L'Action Française, 23. 9. 1929 (Zitate); „A propos de l'Affaire Paul Reynaud“, Ebenda, 24. 9. 1929; "L'affaire Paul Reynaud“, Ebenda, 25. 9. 1929, und öfter.

72 Vgl. etwa: „Bric-à-brac diplomatique“, in: L'Ere Nouvelle, 1. 10. 1929; „Les explications de M. Paul Reynaud“, Ebenda, 10. 10. 1929; "L’impossible alliance“, in: La Volonté, 3. 10. 1929; Léon Blum, "Signe des temps“, in: Le Populaire, 6. 10. 1929.

73 „Une recrue“, in: L'Oeuvre, 10. 10. 1929; „Paul Reynaud a raison“, in: La Volonté, 3. 10. 1929; "Les explications de M. Paul Reynaud“, in: L'Ere Nouvelle, 10. 10. 1929.

74 Vgl. etwa "L'impossible alliance“, in: La Volonté, 3. 10. 1929. 
Zustimmung ernteten die Gespräche - nicht unbedingt die sich abzeichnenden Zukunftsvisionen - vorwiegend in der gemäßigten rechten Presse. Mit einer Ausnahme allerdings: Der nationalistische Publizist Gustave Hervé, der bereits Ansprechpartner Rechbergs gewesen war, betrachtete die Initiative unter dem Aspekt der Annäherung der „Eliten“ beider Länder und der Konstruktion einer antibolschewistischen Bastion in Zentraleuropa als wichtige Alternative zu „pazifistischen" Versöhnungsbemühungen. Zur dauerhaften Fundierung und Ergänzung der Rechbergschen Allianzpläne schlug er den Austausch deutscher linksrheinischer Gebiete gegen Madagaskar und ehemalige deutsche Kolonien vor. ${ }^{75}$

Abgesehen von solchen in Frankreich nicht mehrheitsfähigen Außenseiterinterventionen waren es lediglich die rechtsliberalen Blätter L'Intransigeant und Le Temps, die Reynauds Erkundungsreise offensiv verteidigten. Bezeichnenderweise spielten auch hier kaum projektbezogene Betrachtungen eine Rolle, vielmehr beharrte man in erster Linie auf dem privaten Charakter der Unterredungen, betonte die „Pflicht" führender Politiker, die Annäherungschancen auch mit politischen Gegnern im Ausland auszuloten, und präsentierte Reynauds Vorstoß als Attacke auf das „Monopol“ der Linken im Bereich der Friedenspolitik: „C'est précisément de cela que socialistes et valoisiens ne peuvent s'accommoder. La paix [...] est leur chasse gardée: et c'est de braconnage qu'en définitive ils accusent $M$. Paul Reynaud."76

Was war nun eigentlich geschehen? In der Tat hatte sich Reynaud vom 23. bis 27. April $1929 \mathrm{zu}$ Sondierungsgesprächen in Berlin aufgehalten. Sein Landsmann Pierre Viénot, seit Oktober 1926 Leiter der Berliner Außenstelle des „Comité franco-allemand d'Information et de Documentation " und engagierter Mittler für Kontakte zwischen Vertretern des wirtschaftlichen, politischen und kulturellen Spektrums beider Länder77, war als Verbindungsmann vor Ort tätig geworden

75 Der Gymnasiallehrer und „Agrégé d'histoire“ Gustave Hervé (1871-1944) hatte seine politischen Aktivitäten vor dem Ersten Weltkrieg als Sozialist und militanter Kriegsgegner begonnen und bereits um 1912 die Idee eines deutsch-französischen Ausgleichs über den Austausch von ElsaßLothringen gegen die Insel Madagaskar entwickelt. Mit Kriegsausbruch wandelte er sich zum entschiedenen Patrioten und näherte sich den Ideen Déroulèdes an. 1918 aus der SFIO ausgeschlossen, gründete er den rechtsextremen "Parti Socialiste National“ und trat in seiner Tageszeitung $L a$ Victoire für die Schaffung einer autoritären Republik innerhalb der "natürlichen und historischen Grenzen“ Frankreichs, seit Ende der zwanziger Jahre auch wieder für die deutsch-französische Annäherung ein. Den italienischen Faschismus und aufkommenden Nationalsozialismus betrachtete er mit Sympathie und machte La Victoire ab Juni 1940 zur Verfechterin der Kollaboration mit dem Besatzer (Vgl. zu Hervé mangels einer nach wie vor ausstehenden Gesamtbiographie: Richard Griffiths, Hervé, Gustave, in: Biographical Dictionary of French Political Leaders since 1870, New York u. a. 1990, S. 203 f.; aufschlußreich für seine programmatischen Vorstellungen Ende der zwanziger Jahre ist sein Artikel "Le programme nationaliste“, in: La Victoire, 3. 10. 1929 (Zitat). Seine Ideen für den deutsch-französischen Ausgleich legte Hervé gesammelt dar in: Gustave Hervé, France-Allemagne. La réconciliation ou la guerre, Paris 1931).

76 "L'affaire Rechberg“, in: L'Intransigeant, 2. 10. 1929; „Encore la chasse gardée“, in: Le Temps, 11. 10. 1929 (Zitat).

77 Zur Person Viénots vgl. Hans Manfred Bock, „Connaître l'Allemagne et la reconnaître.“ Zu Entstehung und Zusammenhang der Deutschland-Analyse von Pierre Viénot 1922 bis 1932, in: Lendemains 66 (1992), S. 27-48; Ders., Friedrich Sieburg et Pierre Viénot ou la fin du „Locarno intellectuel“, in: Allemagnes d'aujourd'hui 105 (1988), S. 84-99; Alois Schumacher, Annäherung durch Verstehen. Pierre Viénot und die deutsch-französischen Beziehungen in den zwanziger Jahren, in: Médiations ou le métier de germaniste. Mélanges offerts à Pierre Bertaux, Asnières 1977, S. 271277. 
und hatte ihm zu einem Treffen mit den „bestinformierten“ Franzosen in Berlin, mit Oswald Hesnard 78 und Botschafter Pierre de Margerie verholfen. ${ }^{79}$ Auf Vermittlung dieser beiden Ansprechpartner und Arnold Rechbergs war Reynaud dann im weiteren Verlauf seines Aufenthalts unter anderem mit den Reichstagsabgeordneten Werner von Rheinbaben (DVP), Hartmann von Richthofen (DVP), Gottfried Reinhold Treviranus (DNVP) und Moritz Klönne (DNVP) zusammengetroffen. Weitere Gesprächspartner waren der ehemalige Finanzminister Heinrich Köhler (Zentrum), der Verleger Franz Ullstein sowie der Staatssekretär im Preußischen Staatsministerium, Weismann, ein führender Vertreter des Reichsbanners, Karl Mayr, daneben Kapitänleutnant Ehrhardt, der „Hochmeister" des "Jungdeutschen Ordens" Artur Mahraun, Walter Eberhard von Medem als Vertreter des „Stahlhelm“ sowie Otto Kriegk, ein Mitarbeiter Hugenbergs, gewesen. Auf Empfehlung des Botschafters hin hatten sich schließlich der Leiter des Sonderreferats „Wirtschaftsverhandlungen, Wirtschafts- und Reparationspolitik“ im Auswärtigen Amt, Ritter, sowie Außenminister Stresemann bereit erklärt, Reynaud zu empfangen.

Zumindest im Falle der Gespräche im Auswärtigen Amt sind die angesprochenen Themen eindeutig dokumentiert. Reynaud unterbreitete hier lediglich einen Vorschlag zur Lösung der Reparationsfrage. Die deutschen Verpflichtungen sollten zweigeteilt werden: Den geringeren Teil, der den französischen Wiederaufbaubedürfnissen zugute kommen sollte, wollte Reynaud in eine kommerzielle Schuld umgewandelt wissen. Auf den größeren Teil dagegen, vorgesehen zur Deckung der französischen Schulden gegenüber den USA, war Reynaud bereit zu "verzichten", was er mit politischen Hintergedanken verband: Wenn Deutschland nach einiger Bekundung guten Willens letztlich doch ein Moratorium erbitten müsse, dann würde Frankreich seinerseits seine Zahlungen gegenüber den Vereinigten Staaten suspendieren können. 80

Die deutsche Diplomatie reagierte äußerst zurückhaltend auf die vorgetragenen Ideen. Während das Gespräch mit Stresemann ohnehin auf ganz allgemeiner Ebene blieb und betont als rein "private" Aussprache eingestuft wurde81, hatte sich Ritter in einer deutlich konkreter gehaltenen Unterredung kühl gegeben und war auch auf den Vorstoß Reynauds, „Europa müsse sich gegenüber der wirtschaftlichen Übermacht der Vereinigten Staaten zusammenschließen", nicht weiter eingegangen. ${ }^{82}$ Staatssekretär von Schubert schließlich, der durch den Abgeordneten Klönne über den Besuch Reynauds unterrichtet worden war, hatte sei-

$78 \mathrm{Zu} \mathrm{Hesnard:} \mathrm{Jacques} \mathrm{Bariéty,} \mathrm{Un} \mathrm{artisan} \mathrm{méconnu} \mathrm{des} \mathrm{relations} \mathrm{franco-allemandes:} \mathrm{le} \mathrm{professeur}$ Oswald Hesnard, 1877-1936, in: Media in Francia... Recueil de mélanges offert à Karl Ferdinand Werner à l'occasion de son 65e anniversaire par ses amis et collègues français, Paris 1989, S. 1-18.

$79 \mathrm{Vgl}$. dazu die Briefe Viénots an Reynaud vom 9. 4. und 15. 4. 1929 (Zitat) sowie die Antworten Reynauds vom 9.4. und 17.4. 1929 (AN, 74 AP 11). Zur Reiseorganisation auch: Reynaud an Rechberg vom 5. 4., 19. 4., 22. 4. und 30. 4. 1929 (BAK, NL 49, Dossier 57).

so Aufzeichnung Ritters über ein Gespräch mit Paul Reynaud, 26. 4. 1929 (PA-AA, R 29079 k). Vgl. auch die Niederschrift des Staatssekretärs im Auswärtigen Amt, von Schubert, über einen Bericht Stresemanns zu seinem Gespräch mit Reynaud vom 27.4. 1929 (ADAP B XI, S. 451, Anm. 2). Handschriftliche Aufzeichnung Reynauds über seinen Besuch bei Stresemann, 27. 4. 1929 (AN, 74 AP 11).

81 Niederschrift von Schuberts, 27. 4. 1929, (ADAP B XI, S. 451, Anm. 2).

82 Aufzeichnung Ritters, 26. 4. 1929 (PA-AA, R 29079 k). 
nem Informanten gegenüber deutlich erkennen lassen, daß er das Vorgetragene für nichts weiter hielt als unverbindliche und angesichts der komplexen Problemlage allzu unrealistische Einlassungen. ${ }^{83}$ Die Einschätzung von Schuberts bezog sich besonders auch auf jenen Teil der Vorschläge Reynauds, die dieser bei seinen Gesprächen im Auswärtigen Amt weggelassen und nach Bekunden seiner deutschen Gesprächspartner offenbar nur in privatem Kreise in Gegenwart Rechbergs, Klönnes, von Medems und Kriegks vorgetragen hatte. Hier war nach Aussagen Klönnes auch die Rede von einem deutsch-französischen Militärpakt mit gemeinsamem Generalstab bei einem Kräfteverhältnis von drei zu fünf zugunsten Frankreichs gewesen sowie von der Zusage „freundschaftlichen Drucks“ auf Polen zugunsten einer Restitution des Korridors an Deutschland. ${ }^{84}$

Reynaud verteidigte sich gegen die nach seiner Rückkehr erhobenen Vorwürfe gewandt, ohne indes allzusehr in Details zu gehen. Noch von den Vereinigten Staaten aus nahm er in einem Pressetelegramm zu seiner "Studienreise“ Stellung, hob das Treffen mit Stresemann hervor und erklärte, der ganzen Angelegenheit zu wenig Bedeutung zugemessen zu haben, um davon Mitteilung an die Öffentlichkeit oder seine Abgeordnetenkollegen zu machen. ${ }^{85}$ Nach seiner Rückkehr Anfang Oktober bekräftigte er den rein informatorischen Charakter des Berlinaufenthalts: „Les prétendues négociations se ramènent à un simple voyage d'études que j'ai fait à Berlin au mois d'avril [...]. L'Allemagne officielle est pacifique, mais il y a aussi une minorité fanatique qui pourrait, dans des circonstances conditionnelles, reprendre le pouvoir. J'ai tenu à me rendre compte avec précision du péril. Je voulais voir l'Allemagne complète. J'ai rencontré des hommes de tous les partis, depuis l'extrême gauche jusqu'à l'extrême droite. Tous m'ont accueilli avec une courtoisie que je me plais à reconnaître. Nous avons parlé. Il est facile de transformer des conversations privées en "négociations secrètes. [...]“ 86

Daß er eine zehnprozentige Ermäßigung der nach dem Dawes-Plan festgesetzten Annuitäten in Aussicht gestellt habe - so ein Vorwurf in der Pariser Presse -, bestritt er ebenso wie den Vorschlag einer deutsch-französischen Militärallianz gemäß den Vorstellungen Arnold Rechbergs. Entsprechende Ideen seien nur von deutscher Seite vorgetragen und von ihm unter Hinweis auf die öffentliche Meinung in Frankreich zurückgewiesen worden. Als denkbare Alternative habe er allerdings ein gemeinsames Agieren deutscher und französischer Truppen unter dem Dach des Völkerbunds ins Gespräch gebracht. In der Polenfrage wies Reynaud laut eigenem Bekunden auf die Bündnisverpflichtungen Frankreichs hin und machte eine Lösung der strittigen Grenzfragen von der Einstellung nationalisti-

83 Aufzeichnung von Schuberts, 26. 4. 1929 (ADAP B XI, Nr. 198, S. 449f.) sowie seine zweite Niederschrift vom gleichen Tage (Ebenda, S. 450, Anm. 2).

${ }_{84}$ Aufzeichnung von Schuberts über ein Gespräch mit Klönne, 26.4. 1929 (ADAP B XI, Nr. 198, S. 449).

${ }_{85}$ Das Telegramm wurde veröffentlicht im Rahmen eines Artikels von Marcel Hutin in: L'Echo de Paris, 25. 9. 1929. Die deutsche Botschaft verfolgte die Entwicklung der Kampagne aufmerksam: vgl. etwa Rieth an AA, 25. 9. 1929 (PA-AA, R 70562).

86 L'Echo de Paris, 9. 10. 1929; inhaltsgleich auch in Le Matin, 9. 10. 1929. Vgl. dazu den Bericht von Botschaftsrat Rieth an das Auswärtige Amt vom 9. 10. 1929 (PA-AA, R 29079 k). 
scher Revisionskampagnen in Deutschland und von der Verbesserung der internationalen Lage abhängig. ${ }^{87}$

Diese nachträglich gegebenen, widersprüchlichen Erklärungen der Beteiligten erlauben kein eindeutiges Urteil darüber, ob insbesondere die militärpolitischen Vorschläge Reynauds in der Tat so gemacht wurden, und wie genau die Reaktionen seiner privaten deutschen Gesprächspartner aussahen. Der deutschnationale Abgeordnete Klönne zumindest beharrte ab September 1929 nurmehr auf dem reparationspolitischen Aspekt der Gespräche. Da er wie Reynaud heftigen Presseangriffen ausgesetzt war, die sich vor allem gegen die "doppelte Moral“ der Deutschnationalen und den Widerspruch zwischen offiziell frankreichfeindlichen Äußerungen und vermeintlichen militärischen Bündnisplänen richteten, hatte er allerdings auch ein starkes Interesse daran, eventuelle Gesprächsinhalte dieser oder gebietspolitischer Art im Rückblick geflissentlich zu übergehen. ${ }^{88}$ Auch war Reynaud ganz offensichtlich nicht an einer detaillierten Aufklärung interessiert. Zumindest für den reparationstechnischen Teil der diskutierten Gesprächspunkte kann aber wohl aufgrund der durchaus vertrauenswürdigen Niederschrift Ritters und entgegen Reynauds relativierenden Presseerklärungen davon ausgegangen werden, daß er ganz konkrete Vorschläge unterbreitete, die auf eine klare Verminderung der deutschen Schuld hinausliefen. Auf seiten seiner deutschen Gesprächspartner konnten diese als Aufforderung zur teilweisen Nichtbezahlung unter der Voraussetzung interpretiert werden, daß Frankreichs Wiederaufbaubedürfnisse befriedigt würden.

So realistisch diese Ideen vom Prinzip der Reduzierung der deutschen Reparationslast her sein mochten - die seit Januar 1929 laufenden Expertenberatungen zur Revision des Dawes-Plans verfolgten ja eben jenes Ziel -, so deutlich trat hier doch ein ganz anders argumentierender Reynaud auf als dann in den Debatten zur Ratifikation des Mellon-Bérenger-Abkommens einige Monate später. Stand in seiner öffentlichen Stellungnahme dort der Aspekt der Loyalität gegenüber dem Kriegsalliierten demonstrativ an erster Stelle, so versuchte er in den vertraulich gehandhabten Sondierungsgesprächen vom April 1929, die Möglichkeiten für eine elegante Umschiffung der französischen Verbindlichkeiten mit deutscher Beihilfe auszuloten. Auf inoffiziellem Umwege sollte eine Komplizenschaft zwischen Sieger und Besiegtem begründet werden, die unter moralischem Aspekt nichts weniger als einen illoyalen Akt gegenüber dem überseeischen Gläubiger darstellte. Zwar stand er damit nicht allein, da der Gedanke, mit dem deutschen Reparationsschuldner "gemeinsame Sache" gegen die Vereinigten Staaten zu machen, ab 1928 seitens der französischen Diplomatie wiederholt vertraulich ins Gespräch gebracht worden war. ${ }^{89}$ Es leuchtet aber dennoch ein, daß Reynaud wenig daran

87 Ebenda.

88 Vgl. dazu Klönnes Briefe an Schubert vom 22. 9. 1929 (Köpke an Schubert, 23. 9. 1929; PA-AA R 70562), vom 30. 9. und 11. 10. 1929 (PA-AA, R 29079 k). Zu den Reaktionen der großen deutschen Zeitungen siehe die Analysen der Presseabteilung des Auswärtigen Amts vom 21.9., 22.9. und 23. 9.1929 (PA-AA, R 29079 k).

89 Vgl. Krüger, Außenpolitik, S. 417 f. und 448f. (Zitat: S. 418). Daß Reynaud seinen Vorstoß als Mittelsmann Poincarés unternahm, ist angesichts des wenig vertrauensvollen Verhältnisses zwischen beiden Politikern äußerst unwahrscheinlich; Poincaré wies Vermutungen dieser Art strikt zurück (vgl. dazu das Telegramm Rieth an AA, 25.9. 1929 (PA-AA, R 70562). 
gelegen sein konnte, einen solchen Abspracheversuch an die Öffentlichkeit gelangen zu lassen.

Zweifellos hatte Reynaud im Sommer 1929 klarsichtiger und früher als jene, die noch während der Ratifizierungsdebatten an eine Umgehung der französischen Zahlungsverpflichtung glaubten, die Konsequenzen aus der amerikanischen Unnachgiebigkeit und den Beratungsergebnissen der Young-Kommission gezogen. Anlaß dazu, sich im Rückblick als Verfechter einer moralisch höherstehenden, da am gegebenen Wort gegenüber den USA orientierten Position zu präsentieren, hatte er allerdings kaum, berücksichtigt man seine vorhergegangenen Gespräche in Deutschland. 90

Was andererseits die angeblich durch ihn gemachten militärpolitischen Vorschläge anging - Anregungen, die im übrigen nur indirekt und nicht durch diplomatische Gesprächspartner dokumentiert sind -, so fällt auf, daß diese bis in Details den einschlägigen Ideen Rechbergs entsprechen, ohne dagegen in den Äußerungen Reynauds vorher oder nachher greifbar zu sein.

Arnold Rechberg hatte seine publizistische Kampagne für eine deutsch-französische Annäherung mit der ihm eigenen Hartnäckigkeit seit 1923 unvermindert fortgesetzt. Neben den bekannten Ideen einer wirtschaftlich-industriellen Verflechtung war er ab Mai 1925 in der französischen Presse mit einem modifizierten Konzept an die Öffentlichkeit getreten. Es gab vor, über die Ablösung des Versailler Vertrags durch eine deutsch-französische Militärallianz, die Rückgabe des polnischen Korridors und das französische Einverständnis mit der Angliederung Österreichs die wichtigsten Belastungsfaktoren des Verhältnisses beider Länder mit einem Schlag aus dem Wege zu räumen. Daß er dabei das französische Sicherheitsbedürfnis eklatant unterschätzte, das durch die vorgesehene deutsche Aufrüstung selbst bei einer Kräfteverteilung von „drei zu fünf“ kaum befriedigt worden wäre, gelangte ihm selbst nie wirklich zu Bewußtsein. Unbekümmert auch vom Verlauf und den Ergebnissen der deutschen Locarno-Politik hatte er zur Verbreitung seiner Konzeption in unerschütterlicher, doch letztlich gleichbleibend erfolgloser Aktivität seine Publikationstätigkeit intensiviert und Beziehungen zur Entourage von Reichswehrminister Geßler geknüpft, hatte Kontakte mit dem Auswärtigen Amt gepflegt und im April 1926 und Februar 1929 sogar erreicht, in Paris durch Briand bzw. Poincaré und den damaligen Kriegsminister Painlevé empfangen zu werden. ${ }^{91}$ Erhebliche Verstimmung bei Poincaré hatte er dann jedoch dadurch provoziert, daß er dem an sich recht belanglosen Gespräch im folgenden eine übermäßig weitgehende Ausdeutung unterlegte; die Zustimmung zu einem Militärpakt gegeben zu haben, dementierte der Ministerpräsident in mehreren persönlichen Schreiben vehement. ${ }^{92}$

90 Dies hingegen der Grundtenor seiner Erinnerungen an die Schuldendebatte: Reynaud, Mémoires I, S. 259-273, hier: S. 259 f.

91 Vgl. zu den frankreichpolitischen Aktivitäten Rechbergs zwischen 1927 und September 1929 die ungezeichnete Aufstellung des Auswärtigen Amts: „Übersicht nach dem Stand vom 26. 9. 29“, 26. 9.1929 (PA-AA, R 29079 k) sowie Vietsch, Arnold Rechberg, S. $85 \mathrm{ff}$.

92 Vgl. unter anderem die Briefe Rechbergs an Poincaré vom 12. 10. und 16. 10. sowie die Antworten Poincarés vom 14. 10., 18. 10., 30. 10. und 5. 11. 1929 (Bibliothèque Nationale, N.a.f. 16014, Band XXIII). Dazu auch Vietsch, Arnold Rechberg, S. 113-116. 
Rechbergs notorische Neigung, politische und persönliche Konstellationen über Gebühr zugunsten seines Verständigungsprojekts auszulegen und die eigene Rolle dabei stark zu übertreiben, ließ auch das Verhältnis zu Paul Reynaud nicht unberührt. Dieser stellte für Rechberg zweifellos den wichtigsten politischen Ansprechpartner in Paris dar, mit dem er über Jahrzehnte hinweg - nachweisbar von 1922 bis 1937 - in brieflichem und persönlichem Kontakt blieb. Reynaud war stets auch gemeint, wenn Rechberg von „einflußreichen Kreisen“ in Frankreich sprach, die zur Unterstützung und Verbreitung seiner Ideen bereit seien. Von Beginn seiner Bekanntschaft mit dem französischen Politiker an konnte er jedoch nicht der Versuchung widerstehen, gegenüber deutschen Ansprechpartnern geschönte Angaben zu dessen Stellung im Nachbarland zu machen, ihn als „Privat-Sekretär des Präsidenten Millerand" oder als Verwandten des Industriellen Schneider-Creusot zu charakterisieren. Bereits 1923 schrieb er Reynaud in der französischen Abgeordnetenkammer den Einfluß zu, „den Erzberger während der ersten Kriegsjahre im deutschen Reichstage hatte". .93

Berücksichtigt man diese "autosuggestive Methodik"94 des Deutschen und seine Neigung, Unterredungen im nachhinein einen Sinn zu geben, den sie nicht hatten, so scheint unabweisbar, daß Angaben aus dem Umkreis Rechbergs und der ihm nahestehenden Persönlichkeiten über erzielte „Verhandlungsergebnisse“ mit französischen Gesprächspartnern nur mit höchster Vorsicht zu betrachten sind. Reynaud jedenfalls dementierte verärgert in einem an Rechberg gerichteten privaten Schreiben vom 23. November 1929, bei seinem vergangenen Berlinbesuch von einer Militärallianz im Rechbergschen Sinne gesprochen zu haben. ${ }^{95}$

Ganz abgesehen von der genauen Tragweite der gemachten Äußerungen hielten sich die Erfolgsaussichten der Kontaktversuche von vorneherein in sehr engen Grenzen. Zwar hatte Rechberg seinen französischen Gast durchaus mit einem interessanten, überparteilichen Querschnitt von Persönlichkeiten in Verbindung gebracht, denen eine frankreichfreundliche Haltung unterstellt werden konnte. Daß Gespräche mit Vertretern der Linken wie dem bekanntermaßen frankophilen Rudolf Breitscheid oder selbst einem führenden Mann im „Reichsbanner SchwarzRot-Gold“ wie dem ehemaligen bayerischen Generalstabsoffizier Mayr von vorneherein auf der Ebene des unverbindlichen Gedankenaustausches bleiben würden, lag auf der Hand.96 Wenn jedoch, wie Reynaud und Rechberg im nachhinein

93 Vgl. Rechberg an Stinnes, 29. 1. 1923; Ausarbeitung Rechbergs zum Frankreichproblem, 12. 1. 1923 (PA-AA, R 28189 k). Der Karton enthält eine umfangreiche Dokumentation der publizistisch-politischen Aktivitäten Rechbergs.

Die Ansicht, daß Reynaud Schwiegersohn Schneider-Creusots sei, nahm von hier ihren Ausgang und setzte sich sowohl in der zeitgenössischen deutschen Diplomatie als auch in der neueren historischen Forschung fest: Vgl. die Aufzeichnung von Bülows vom 14.2.1931 und die Richtigstellung von Hoeschs, 10.3. 1931 (PA-AA, R 70563); Wulf, Stinnes, S. 361 f.; Piotr S. Wandycz, The Twilight of French Eastern Alliances, 1926-1936. French-Czechoslovak-Polish Relations from Locarno to the Remilitarization of the Rhineland, Princeton 1988, S. 139.

94 Vietsch, Arnold Rechberg, S. 115.

95 Ebenda, S. 116.

96 Zu Breitscheid und Frankreich: Marie-Dominique Cavaillé, Rudolf Breitscheid et la France, 19191933, Frankfurt a.M. u.a. 1995; zu den Konzeptionen Mayrs einer internationalen, antibolschewistischen „Frontkämpferpolitik“ auf der Grundlage der deutsch-französischen Aussöhnung vgl. Karl Rohe, Das Reichsbanner Schwarz-Rot-Gold. Ein Beitrag zur Geschichte und Struktur der 
ausführten, das Ziel der Kontakte vor allem in der Annäherung verständnisbereiter Kreise des „nationalen Milieus“ beider Länder bestand, dann lagen dafür im April 1929 nurmehr sehr ungünstige Voraussetzungen vor.

Zweifellos hatte es im "Jungdeutschen Orden“, der neben dem "Stahlhelm" gewichtigsten antirepublikanischen rechten Frontkämpfervereinigung, auf Betreiben ihres Gründers und „spiritus rector“ Artur Mahraun seit 1925 Bestrebungen gegeben, die bis dahin frankophobe Grundhaltung des Verbandes durch eine flexiblere Position zu ersetzen. Der Inspirator hierfür war in hohem Maße Arnold Rechberg gewesen, dessen Einfluß auf den „Ordensgründer" $z u$ einer zeitweisen Zusammenarbeit geführt hatte. Auf Rechbergs Vermittlung hin hatte Mahraun im Herbst 1925 seine Ideen für eine deutsch-französische wirtschaftliche Verflechtung als Voraussetzung zur Aufhebung des Versailler Vertrags und die Revision der deutschen Ostgrenze gegenüber dem Direktor des konservativen Le Matin, Jules Sauerwein, ausbreiten können. Vorwiegend motiviert durch eine interne ideologische Neuorientierungskrise des Verbands, war die neue "Westpolitik“ zu Jahresbeginn 1929 offiziell jedoch bereits wieder zugunsten einer dogmatischen Haltung aufgegeben worden.97

Im nationalen Milieu hatte der "Jungdo“ zudem mit diesem wenig fundierten Vorstoß von Anbeginn eine weitgehende Ausnahmestellung innegehabt. Wenn Arnold Rechberg geglaubt hatte, in den Reihen des „Stahlhelm“ die Bereitschaft $\mathrm{zu}$ ähnlichem Engagement entdeckt $\mathrm{zu}$ haben, und sich während seines Parisaufenthalts vom Frühjahr 1929 als autorisierter Sprecher darstellte, so verriet die Episode wiederum mehr über das Wunschdenken des Amateurpolitikers als über tatsächlich vorhandene Ausgleichswilligkeit in den Reihen der Frontkämpfervereinigung. ${ }^{98}$ Auch in den Führungsgremien der DNVP war man nicht gewillt, die Partei in Verbindung mit den Frankreichkontakten einzelner ihrer Mitglieder bringen zu lassen. ${ }^{99}$ Im Jahre 1929 reichten weder der Einfluß Rechbergs noch die grundsätzlichen Dispositionen in den wichtigsten rechten Gruppierungen hin, um als Basis für eine realistische Verständigung zu dienen.

Diese gravierende Fehleinschätzung des Möglichen disqualifizierte Rechberg als Vermittler von Gewicht, zumal seine wiederholten Vorstöße - von denen sich ja die kolportierten „Vorschläge“ Reynauds nicht wesentlich unterschieden hätten - in den maßgeblichen politischen und diplomatischen Kreisen beider Länder zu

politischen Kampfverbände zur Zeit der Weimarer Republik (Beiträge zur Geschichte des Parlamentarismus und der politischen Parteien 34), Düsseldorf 1966, S. 149-152, Zitat: S. 149.

97 Grundlegend zur „Frankreichpolitik“ des Jungdeutschen Ordens: Dieter Tiemann, Der Jungdeutsche Orden und Frankreich, in: Francia 12 (1984), S. 425-456; allgemein zum Jungdo: Klaus Hornung, Der Jungdeutsche Orden (Beiträge zur Geschichte des Parlamentarismus und der politischen Parteien 14), Düsseldorf 1958, hier: S. 42-50. Über das Verhältnis Rechbergs zum Jungdeutschen Orden auch: Vietsch, Arnold Rechberg, S. 96-99.

98 Der "Stahlhelm" wehrte sich sogar erfolgreich vor Gericht gegen Behauptungen des konkurrierenden "Jungdeutschen Ordens", mit den Verständigungsbemühungen Rechbergs in Verbindung zu stehen; vgl. Volker R. Berghahn, Der Stahlhelm. Bund der Frontsoldaten 1918-1935 (Beiträge zur Geschichte des Parlamentarismus und der politischen Parteien 33), Düsseldorf 1966, hier: S. 156-158.

99 Vgl. hierzu den Tagebucheintrag des führenden DNVP-Abgeordneten Reinhold Quaatz vom 23. 9. 1929, in: Hermann Weiß/Paul Hoser (Hg.), Die Deutschnationalen und die Zerstörung der Weimarer Republik. Aus dem Tagebuch von Reinhold Quaatz 1928-1933 (Schriftenreihe der Vierteljahrshefte für Zeitgeschichte 59), München 1989, S. $81 \mathrm{f}$. 
diesem Zeitpunkt nicht mehr ernst genommen wurden. ${ }^{100}$ Der deutsche Botschafter in Paris, von Hoesch, hatte Rechberg nach eigenem Bekunden „als Erkundungsreiter manchmal ganz praktisch gefunden“, überdies aber genügend Anlaß gehabt, sich über dessen „unglückselige Schriftstellerei in der Pariser Presse“ zu beklagen. ${ }^{101}$ Sein französischer Kollege in Berlin, de Margerie, schrieb Rechberg "mehr Einbildungskraft als Realitätssinn" 102 zu. Poincaré selbst hatte nach einschlägigen Auskünften Hoeschs ${ }^{103}$ und besonders nach den erwähnten Interpretationsstreitigkeiten keinen Grund mehr, den Deutschen als Gesprächspartner von Belang einzuschätzen. Außenminister Stresemann schließlich distanzierte sich im Frühsommer 1929 wie die übrige deutsche Diplomatie zum wiederholten Male von den Vorstössen Rechbergs und ließ Hoesch mitteilen, daß die Vorschläge des Industriellen als „für absehbare Zeit undiskutierbar" einzustufen seien.104 Die Bedenken der deutschen Diplomatie galten besonders den unabsehbaren Auswirkungen eines Militärbündnisses auf die Beziehungen Deutschlands zu Großbritannien, der Sowjetunion und den Vereinigten Staaten, außerdem erachtete man den Verzicht Frankreichs auf seine militärische Überlegenheit als äußerst unwahrscheinlich, ganz zu schweigen von französischer Hilfe zur Revision der deutschen Ostgrenze. 105

Der Gegensatz zwischen dem fehlenden wirklichen Einfluß Rechbergs und seinem vollmundigen Auftreten blieb Reynaud nicht verborgen und führte zu einer entscheidenden Abkühlung des beiderseitigen Verhältnisses. Den konkreten Anlaß hierzu bildete eine Presseverlautbarung des „Stahlhelm“, aus der das Beharren der Organisation auf dogmatischen antifranzösischen Positionen und insbesondere auf der Leugnung jeder deutschen Reparationsverpflichtung gegenüber

100 Den kaum vorhandenen Einfluß Rechbergs auf die deutsche Politik überschätzt: Maarten L. Percboom, Democracies at the Turning Point. Britain, France and the End of the Postwar Order, 19281933 (Studies in Modern European History 13), New York u. a. 1995, S. 149 f. (Zitat: S. 149). Pereboom versteht Rechberg als "one of the driving forces" hinter der deutschen Bereitschaft zur internationalen wirtschaftlichen Kooperation, wie sie sich etwa im deutsch-französischen Kaliabkommen vom Dezember 1926 oder dem Handelsvertrag vom August 1927 niederschlug. Zweifellos war Rechberg ein öffentlich wirksamer Befürworter solcher Verflechtungsansätze, doch läßt sich irgendwelcher Einfluß auf den Gang der Verhandlungen nicht nachweisen. Entsprechende Vermutungen in der zeitgenössischen Öffentlichkeit beruhten zum Teil auf einer Verwechslung seiner Person mit seinem Bruder, dem Kali-Industriellen Fritz Rechberg (Vietsch, Arnold Rechberg, S. 92-95).

$10 t$ Hoesch an AA, 27. 1. 1927; Hoesch an Stresemann, 28. 2. 1929 (PA-AA, R 70562).

$102 \mathrm{Vgl}$. die Telegramme von Botschafter de Margerie an Briand vom 27. 7. 1925 (MAE, Europe 19181940, Allemagne, Band 343), 24. 4. 1926 (Ebenda, Band 389), 19. 6. 1928 (Ebenda, Band 391)(Zitat) und 9. 2. 1929 (Ebenda, Band 392).

Zur Einschätzung im Quai d'Orsay auch: Bericht des Militärattachés der französischen Botschaft Berlin, Colonel Tournès, über ein Gespräch mit Rechberg, 9. 12. 1927 (Ebenda, Band 390); Note der "Direction politique et commerciale“ "A.s. prétendues négociations entre "nationalistes" français" et allemands“, 28. 9. 1929 (Ebenda, Band 393); den Einfluß Rechbergs in Deutschland überschätzt: Bericht der Sûreté über Arnold Rechberg, 22. 5. 1929 (Ebenda, Band 392).

103 Bericht Hoesch über eine Aussprache mit Poincaré und Kriegsminister Painlevé zu deren Unterhaltungen mit Rechberg, 28. 2. 1929 (PA-AA, R 70562).

10+ Von Bülow an Botschaft Paris, 27. 5. 1929 (PA-AA, R 70562)(Zitat); Stresemann an Botschaft Moskau, 1. 6. 1929 (Ebenda); vgl. auch das auf Betreiben Staatssekretär von Schuberts entstandene offizielle Dementi des Auswärtigen Amts, über die Gespräche Klönne-Rechberg-Reynaud unterrichtet gewesen zu sein: Kaufmann (Genf) an AA, 23.9.1929 (PA-AA, R 29079 k).

105 Vgl. dic Stellungnahme des Staatssekretärs im Auswärtigen Amt, von Schubert, zu den Plänen Rechberg/Reynauds (ADAP B XI, Nr. 198, S. 449-451). 
Frankreich hervorging. Die Tatsache, daß die Erklärung nur wenige Tage nach der Unterredung Reynauds mit einem seiner führenden Mitglieder erfolgt war, befremdete den Politiker, zumal Rechberg auf Nachfrage hin ausweichend reagierte und als schwachen Erklärungsversuch unter anderem „bolschewistische Agenten" ins Feld führte, die nach dem Berlinbesuch Reynauds eine Allianz deutscher und französischer Konservativer verhindern wollten. ${ }^{106}$ So fiel die Warnung, die Pierre Viénot seinem Landsmann Reynaud hinsichtlich allzugroßen Vertrauens in die Vermittlung Rechbergs und die Verständigungsbereitschaft seiner rechtsstehenden Freunde auf den Weg gab, auf bereits vorbereiteten Grund: „Vous savez $[\ldots$. ] que j'ai très grande confiance, et les relations personnelles les plus sûres, dans des Allemands appartenant nettement à la droite. Mais il faut distinguer, et je ne puis que vous redire ici, après une longue expérience et des tentatives répétées de collaboration avec les milieux mêmes au sein desquels Reichberg[!] vous a fait pénétrer, mon peu de confiance dans la possibilité d'un accord avec ces extrémistes." 107

Das Wort Viénots, der zu diesem Zeitpunkt bereits von der „Lernfähigkeit“ der sein Komitee tragenden gemäßigten Rechten in beiden Ländern tief enttäuscht war ${ }^{108}$, zeigte Wirkung. Zwar bedeutete die Ernüchterung Reynauds noch nicht den Abbruch aller „persönlichen Beziehungen“ zwischen den beiden Männern. 109 Doch verlief der Meinungsaustausch, der ab dann vorwiegend auf Anschreiben Rechbergs hin aufrechterhalten wurde, nach einer kurzen Wiederaufnahme des Kontakts im Herbst 1929 allmählich im Sande. ${ }^{110}$

Zunächst noch unberührt von dieser auf lange Sicht wirksamen Entfremdung Reynauds von seinem bevorzugten Ansprechpartner in Deutschland blieb sein deutschlandpolitisches Konzept, das er in Reaktion auf die zumeist innenpolitisch motivierte Kritik in einer Kammerdebatte im Dezember 1929 offensiv vertrat. So überraschend Reynauds Deutschlandreise für die nicht informierte Öffentlichkeit gewesen und so erfolglos sie letztlich geblieben war, so sehr entsprang sie doch einem deutschlandpolitischen Interesse, das sich auf weniger spektakuläre Weise seit 1923 kontinuierlich manifestiert hatte.

\section{b) Zur Fortentwicklung der deutschlandpolitischen Ideen Reynauds seit 1923}

Bis Anfang 1924 hatte das deutschlandpolitische Engagement Paul Reynauds keine greifbaren Erfolge gezeitigt. Es hatte ihn im Gegenteil weiten Teilen der eigenen parlamentarischen Mehrheit suspekt gemacht als etwas eigensinnigen, unberechenbaren Außenseiter, der mit seiner Kritik am Mehrheitsführer und Ministerpräsidenten zwar ein latent vorhandenes Gefühl des Ungenügens an dessen Politik aufgegriffen, sich in seinen Alternativvorschlägen aber gefährlich nahe an Ideen der linken Opposition angenähert hatte. Die anstehenden Neuwahlen leg-

106 Rechberg an Reynaud, 11. 5. 1929; Reynaud an Rechberg, 7. 5. 1929 (AN, 74 AP 11).

107 Viénot an Reynaud, 29. 4. 1929 (AN, 74 AP 11).

${ }_{108}$ Bock, Connaître l'Allemagne, S. 42 f.(Zitat S. 42).

109 Dies behauptet Vietsch, Arnold Rechberg, S. 117.

110 Siehe hierzu den Briefwechsel im Dossier "Arnold Rechberg. Correspondance 1929-31" des Nachlasses Reynauds (AN, 74 AP 11) sowie: Reynaud an Rechberg, 23.11. 1929 (BAK, NL 49, Dossier 57). 
ten Reynaud eine gewisse Mäßigung nahe, und in der Tat bemühte er sich, in der Folge an seiner Loyalität gegenüber Poincaré und dem „Bloc National“ keinen Zweifel zu lassen. So spielte die Deutschlandfrage in seinem Wahlkampf vom Mai 1924 kaum eine Rolle gegenüber dem allesbeherrschenden, innenpolitisch ausgerichteten Schlagabtausch mit dem sich abzeichnenden Linkskartell. Nach seiner Niederlage um die Erneuerung des Parlamentsmandats waren es dann der resultierende Verlust an Einfluß und Artikulationsmöglichkeit, die Rückkehr in den Anwaltsberuf und natürlich die Sorge um die Wiederwahl, die die Beschäftigung mit anderen Themen nahelegten. Für persönliche Vermittlungsaktionen jedenfalls standen die Zeichen zunehmend ungünstiger, als nach dem Antritt des Linkskartells unter Herriot die Idee des Ausgleichs mit Deutschland zur offiziellen Maxime französischer Außenpolitik avancierte.

Nicht allein die Vorbehalte gegen den nun deutschlandpolitisch agierenden innenpolitischen Gegner waren indes ausschlaggebend, wenn sich Reynauds Einschätzung der Beziehungen mit dem östlichen Nachbarn und ihrer Entwicklungsmöglichkeiten in der Folgezeit allmählich verdüsterte. Zunehmende Sorge bereitete ihm inbesondere das militärische Kräfteverhältnis zwischen Frankreich und Deutschland: Angesichts drohender Pläne des Linkskartells zur Wehrzeitverkürzung und Mißständen in der Armee sah er es als extrem gefährdet an, falls es nicht gelänge, eine mobile und rasch einsetzbare Offensivtruppe zur Ahndung deutscher Vertragsverletzungen zu schaffen. ${ }^{11}$ Die langsam außer Kontrolle geratende französische Finanzkrise in Verbindung mit der demographischen und industriellen Überlegenheit Deutschlands, das allmähliche Entgleiten materieller Sicherheitsgarantien nach dem im August 1924 durch Herriot zugesagten Ruhrabzug, schließlich die seiner Meinung nach absehbar vorgezogene Evakuierung der ersten Rheinlandzone ließen Reynaud im Mai 1925 eine bestürzte und für seine Verhältnisse ungewohnt pessimistische Bilanz ziehen. Weder der Institution des Völkerbundes noch dem Genfer Protokoll vom Oktober 1924 oder den seit Frühjahr 1925 laufenden Verhandlungen um eine englische Sicherheitsgarantie für die Rheingrenze traute er angesichts des angloamerikanischen Unverständnisses für französische Bedrohungslagen die Kapazität zur Lösung der Sicherheitsprobleme zu. „Occupation provisoire de la Rhénanie, désarmement de l'Allemagne, alliance avec les petites nations de l'Europe centrale, protocole, pacte, influence morale de la Société des Nations, tel est le clavier sur lequel jouent nos hommes d'Etat. Nous avons vu qu'à l'analyse, aucune de ces garanties ne nous apporte une certitude de sécurité. Nous n'avons su faire à fond, depuis l'armistice, ni une politique réaliste, ni une politique pacifiste." 112

Merkwürdig ratlos und augenscheinlich ohne wirkliche Alternativvorstellungen zu den beiseite gelegten Optionen zog sich Reynaud auf eine fatalistisch getönte Zukunftsprognose zurück: „Le destin de la France rivée au flanc de la prolifique Allemagne est, pour longtemps peut-être, de „vivre dangereusement“.“ Ein-

111 Paul Reynaud, „Avons-nous l'armée de nos besoins ou l'armée de nos habitudes?“, in: Revue Hebdomadaire, 5. 7. 1924, S. 5-11.

112 Paul Reynaud, „Le rêve de la sécuritéc, in: Ebenda, 20. 6. 1925, S. 304-315 (Zitat: S. 315). Der Artikel entstand aus einem Vortrag, den Reynaud am 24. Mai 1925 vor der „Assemblée générale de la Société des Officiers de complément" gehalten hatte. 
ziger Hoffnungspol: Die moralische Festigung der Nation nach dem Vorbild des antiken Griechenland oder Preußens im 18. Jahrhundert, Staatengemeinschaften also, denen es letztlich gelang, gerade wegen ihrer gefährdeten Lage die Oberhand über den übermächtigen Gegner zu behalten: „Peut-être notre peuple, épuisé par la plus sanglante des guerres, se laisserait-il glisser à la décadence s'il vivait dans la sécurité totale d'une péninsule lointaine. L'alerte dans laquelle nous fait vivre notre position géographique, le stoïcisme qu'elle nous impose, les apports qu'elle nous oblige à accepter de peuples divers, n'est-ce pas l'un des facteurs décisifs d'une histoire si riche de passé et plus riche encore de promesses! Refaisons la cohésion morale de ce peuple magnifique. Rendons-lui la fierté évanouie de la victoire [...]. Durcissons son âme, exaltons-la. “113

Geistreiche Koketterie oder realistische Einschätzung von Machtverschiebungen, die in der historischen Wissenschaft Jahrzehnte später als das „Ende der französischen Vorherrschaft in Europa "114 bezeichnet werden würden? Mit dem seismographischen Gespür des informierten Beobachters hat Reynaud in der Tat entscheidende Veränderungen in den internationalen Staatenbeziehungen seit der Verabschiedung des Dawes-Plans erkannt, die den Handlungsspielraum der französischen Führung gegenüber Deutschland zunehmend verringerten. Das französische Eingehen auf die deutsche Sicherheitsinitiative vom Februar 1925, die in der Folge zu den Vereinbarungen von Locarno führte, entsprang ja letztlich eben der Einsicht, daß es Frankreich nicht mehr möglich war, eine „autonome Sicherheitslösung" aus eigener Kraft durchzusetzen. ${ }^{115}$ Mit Aufatmen und Resignation ob der zu leistenden politischen Opfer reagierte Reynaud auf die Locarno-Verträge: beklagte er doch das definitive Ende der Rheinlandbesetzung und die Lockerung der französisch-polnischen Allianz gegen militärische Beistandsversprechen Großbritanniens und Italiens von zweifelhaftem Wert: „Faut-il regretter l'accord de Locarno? Non, il faut féliciter M. Aristide Briand de l'avoir conclu. Il faut seulement le supplier d'en montrer à l'opinion française les possibilités, les limites et les dangers. Cette politique était devenue la seule possible depuis qu'en septembre 1923, au lendemain de la fin de la résistance passive, le gouvernement français d'alors avait laissé passer l'occasion de gagner la paix, depuis qu'à Londres, en juillet 1924, M. Herriot avait abandonné les gages de la France sans aucune contrepartie, ni pour l'exécution du plan Dawes, ni pour la sécurité, ni pour le traité de commerce avec l'Allemagne ". 116

Reynauds nüchterne und klarsichtige Interpretation basierte auf der Berücksichtigung zweier struktureller Momente, die in Zukunft Konstanten seines deutschlandpolitischen Denkens bilden sollten. Illusionslos leitete er die Notwen-

113 Beide Zitate: Ebenda, S. 315.

114 Vgl. Stephen A. Schuker, The End of French Predominance in Europe. The Financial Crisis of 1924 and the Adoption of the Dawes Plan, Chapel Hill 1976.

115 Knipping, Ende der Locarno-Ära, S. 17 f. Stellvertretend für die umfangreiche Literatur zur Vorgeschichte der Locarno-Verträge sei hier genannt: Jon Jacobson, Locarno Diplomacy. Germany and the West, 1925-1929, Princeton 1972; neuerdings auch im Überblick: Klaus Hildebrand, Das vergangene Reich. Deutsche Außenpolitik von Bismarck bis Hitler 1871-1945, Stuttgart 1995, S. $452-460$.

116 Paul Reynaud, „Locarno et Amiens“, in: Revue Hebdomadaire, 31. 10. 1925, S. 608-611 (Zitat: S. 610). 
digkeit zur Fortsetzung der deutsch-französischen Annäherungspolitik aus der machtpolitischen Unfähigkeit Frankreichs her, eine Störung der europäischen Ordnung durch den deutschen Nachbarn aus eigener Kraft und ohne Beistand aus der angloamerikanischen Welt zu parieren. Die Schwächen der Locarno-Vereinbarungen, insbesondere das Fehlen einer französisch-britischen Militärkonvention, bildeten künftig einen konstitutiven Teil seiner diesbezüglichen Argumentation. ${ }^{117} \mathrm{Daß}$ die atmosphärische Veränderung im deutsch-französischen Umgang seit Locarno ein Beharren Frankreichs auf restriktiven Positionen in der Räumungs- oder auch der Entwaffnungsfrage unter verstärkten „Begründungszwang" stellte, war ihm ebenfalls klar; daß damit der revisionspolitische Handlungsspielraum Deutschlands entscheidend erweitert worden war, floß als weitere Konstante in seine Lagebeurteilung ein. ${ }^{118}$

\section{c) Französische Deutschlanddebatten im Zeichen deutschen Revisionsstrebens: die konzeptionelle Annäherung Reynauds an die Vorstellungen André Tardieus}

Ausgelöst durch die Konstituierung der Regierung Tardieu, entbrannte Anfang November 1929 in der französischen Kammer eine außenpolitische Grundsatzdebatte, die in der Weihnachtswoche erneut aufflammte. Reynaud nutzte sie, um dem Regierungschef als einer der Meinungsführer des rechtsliberalen Spektrums im Namen der Kammergruppe „Action démocratique et sociale“ beizuspringen. Dies fiel ihm um so leichter, als daraus die Gelegenheit erwuchs, sein deutschlandpolitisches Konzept mit neuem Gewicht zu Gehör zu bringen, die umstrittenen Gesprächskontakte vom April einzubetten und sich nicht zuletzt für höhere Aufgaben zu empfehlen.

Den Kern der Diskussionen machten die Bestimmungen des Young-Plans, die Rheinlandräumung und die Verhandlungen zur Regelung der Saarfrage aus; den Hintergrund dazu gab das verstärkte deutsche Revisionsstreben ab, das die Kammer mit Bestürzung zur Kenntnis nahm. ${ }^{119}$ So stand insgesamt nichts weniger als die Fortsetzung der Außenpolitik Briands zur Debatte. Die Ergebnisse der Haager Konferenzen hatten Kritik vor allem seitens der Rechten hervorgerufen, während man in den Reihen der sozialistischen wie der radikalsozialistischen Linken den gefundenen Kompromissen ganz überwiegend zustimmte. Man begrüßte darin die Chance auf weitere Verständigung mit dem deutschen Nachbarn oder beugte sich schlicht der Logik der einmal eingeleiteten Entwicklung. Die Rechte hingegen verurteilte Briands Politik als Beleg fehlender Klarsichtigkeit. In sich widersprüchlich, schwanke sie zwischen der Erhaltung des Versailler Systems und Ansätzen zur Verständigung, wodurch immer neue deutsche Forderungen eher ermuntert als eingedämmt würden. ${ }^{20}$ Die Rolle eines Wortführers dieser Tendenz

117 So etwa in seiner großen deutschlandpolitischen Kammerrede vom 24. Dezember 1929 (JO, Chambre des Députés, Débats parlementaires, $2^{e}$ séance du 24 décembre 1929, S. 4641 f.).

118 Als grundlegende Analyse der deutschlandpolitischen Problemlagen Frankreichs gegen Ende der zwanziger Jahre vgl. Knipping, Ende der Locarno-Ära, S. 9-31, hier bes.: S. 17-21 (Zitat: S. 19).

119 Zur Verhärtung im deutsch-französischen Verhältnis seit Herbst 1929: Knipping, Ende der Locarno-Ära, S. 94-96.

120 Bonnefous, Histoire IV, S. 378 f. Als bezeichnende Stimme aus dem rechten Lager vgl. die Haltung 
spielte ein Mann der Mitte, der radikalsozialistische Dissident Franklin-Bouillon. Gegner der Ratifizierung des Young-Plans und des Abzugs aus dem Rheinland, nahm er in durchaus eindrucksvoller Weise die Steigerung des deutschen Wehretats als Ausgangspunkt für grundsätzliche Kritik an einer Politik der fortwährenden Kapitulationen vor Deutschland: Sie beruhe letztlich auf einem seit Locarno zu Unrecht genährten, „tragischen Mißverständnis“ über den deutschen Willen zum Ausgleich. ${ }^{21}$

Reynaud griff am 24. Dezember in die Debatte ein. Der Aspekt der Sicherheit Frankreichs nach der Aufgabe des letzten physischen Faustpfands gegenüber Deutschland stand im Mittelpunkt seiner Ausführungen. Sie waren als Verteidigung der erst wenige Wochen vorher eingeleiteten Regierungspolitik konzipiert. In Antwort vorwiegend auf Franklin-Bouillon stellten sie gleichzeitig ein realpolitisch akzentuiertes Bekenntnis zu knapp fünf Jahren "Locarno-Politik“ dar.122 Es ging Reynaud darum, den eingeschränkten Wert der drei traditionellen Säulen französischen Sicherheitsdenkens seit Versailles - Rheinlandbesetzung, Entwaffnung, Allianzen - zu demonstrieren, um die Fortsetzung des durch Briand eingeschlagenen Wegs der deutsch-französischen Annäherung einsichtig zu machen. Reynaud gehörte zu den Anhängern des vorzeitigen Abzugs französischer Truppen aus den letzten Besatzungszonen: aus sachlichen Gründen einerseits, da er unter Berufung auf Äußerungen Fochs den tatsächlichen militärischen Nutzen bezweifelte; aus ostentativer Loyalität gegenüber Poincaré andererseits, unter dessen Ministerpräsidentschaft Frankreich das Genfer Protokoll vom September 1928 unterzeichnet hatte, das den Abzug vorsah, sobald eine einvernehmliche Regelung der Reparationsfrage gefunden wäre.123 Die kausale Verknüpfung zwischen Young-Plan und Rheinlandabzug legte er anders als die Gegner Briands als bindend zugrunde. Würde Frankreich seine Zusage zurückziehen, brüskiere es sämtliche Mitunterzeichner und begebe sich freiwillig in die diplomatische Isolation: „Si la France agissait ainsi, elle dresserait en face d'elle non seulement l'Allemagne, l'Amérique, l'Angleterre et la Belgique, mais tous les signataires, tous les bénéficiaires du plan Young détruit par ce geste." 124

Überzeugt vom Willen zur Wiederaufrüstung selbst in den Reihen der deutschen Linken, stellte er dem nicht ausschließbaren militärischen Erstarken des östlichen Nachbarn die geringe Verläßlichkeit des eigenen Bündnissystems gegenüber: Angesichts des Wegfalls des russischen Verbündeten, des Rückzugs der USA auf den amerikanischen Kontinent, der englischen Vorbehalte, sich auf dem europäischen Kontinent zu binden und des begrenzten Potentials der östlichen Verbündeten, so Reynaud, hätten die Kategorien traditionellen Gleichgewichts-

de Wendels gegenüber der Politik Briands: Jeanneney, de Wendel, S. 425 f.; Hagspiel, Verständigung, S. 436-456; Siebert, Aristide Briand, S. 530-534.

121 Vgl. seine Debattenstellungnahmen in: JO, Chambre des Députés, Débats parlementaires, Séance du 21 novembre 1929, S. 3401 ff.; Ebenda, Séance du 23 décembre 1929, S. 4615 ff. (Zitat). Dazu auch Hagspiel, Verständigung, S. 453-456; Siebert, Aristide Briand, S. 566.

122 JO, Chambre des Députés, Débats parlementaires, 2c séance du 24 décembre 1929, S. 4640-4645. Irreführend Hagspiel, Verständigung, S. 455, Anm. 113, der als Bekräftigung der Kritik FranklinBouillons eine Redepassage Reynauds zitiert, die sich gerade gegen jenen richtete.

123 Ebenda, S. 4640.

124 Ebenda. 
denkens in Europa nurmehr begrenzte Gültigkeit. ${ }^{125}$ Die im April persönlich praktizierte Kontaktaufnahme auf breiter Basis erhob er deshalb zum Programm, mit charakteristischer Absetzung allerdings von entsprechenden Konzepten der Linken. Statt des realitätsfernen Topos von den "deux Allemagnes“ - unbrauchbar seiner Meinung nach zur Erfassung der komplexen Realität des Nachbarlands plädierte er für eine pragmatische Politik. Pazifistische Zirkel alleine böten in Deutschland eine viel zu schmale Basis für die tragfähige Annäherung: „Il n’y a pas une bonne et une mauvaise Allemagne. Il n'y a pas une éternelle Allemagne. Il y a une Allemagne moderne infiniment complexe et qui évolue tous les jours. Le premier de nos devoirs est de prendre contact avec cette Allemagne, de l'étudier, de la comprendre, d'essayer de dissocier les éléments hostiles à la politique de paix. [...] 'On ne fait d'incursions qu'en pays ennemi', disait M. Thiers." ${ }^{126}$

$\mathrm{Da}$ dem vorgetragenen Programm auch eine deutliche Spur geschickter Apologie in eigener Sache innewohnte, ist evident. Abgesehen davon aber legte Reynaud im Dezember 1929 ein durchaus selbständiges Konzept vor. Es transponierte - weit entfernt von den eher grobschlächtigen Analysen Arnold Rechbergs - eigene Ansätze aus den frühen zwanziger Jahren mutatis mutandis in die veränderte Gegenwart der Nach-Locarno-Ära und der deutschlandpolitischen Neuorientierung unter André Tardieu. Bezeichnend ist, daß ihm die Idee der wirtschaftlichen Verflechtung beider Länder, die er sechs Jahre zuvor propagiert hatte und die seither in bilateralen Industrievereinbarungen wesentlich vorangetrieben worden war, nicht mehr ausreichend erschien, um eine stabile Friedensordnung in Europa zu begründen. Das Politische hatte dem Wirtschaftlichen den Weg zu bereiten, und nicht mehr umgekehrt, denn: „Vous ne pouvez pas songer à rationaliser l'Europe, à faire une répartition de la production entre les pays d'Europe si vous ne donnez pas d'abord à chacun de ces pays l'impression de la sécurité." 127 Diese Neubewertung der Sicherheitsfrage und der damit verbundene, seit 1924 bereits sich abzeichnende deutschlandpolitische Paradigmenwechsel im Denken Reynauds spiegelt nicht nur den Bedeutungszuwachs wider, den die Sicherheitsproblematik insgesamt in den Überlegungen weiterer Kreise von Parlamentariern, verantwortlichen Politikern und in der politischen Öffentlichkeit Frankreichs gewonnen hatte. Der Wandel der Prioritäten auch und gerade in den Konzeptionen des Wirtschaftsfachmanns Reynaud zeigt darüber hinaus eine in der moderneren Forschung thematisierte Einsicht als bereits zeitgenössische: die Abhängigkeit der wirtschaftlichen Annäherungsbemühungen der Jahre nach Locarno von der Gunst der politischen Rahmenbedingungen.128 Reynaud formulierte sie als Schlußfolgerung aus dem Stocken der wirtschaftlichen Verständigungsansätze seit 1925. Er markierte damit zugleich die Tendenz in den politisch-parlamentarischen Führungszirkeln Frankreichs, die Politik des internationalen Ausgleichs wieder stärker dem nationalen Interesse unterzuordnen. Zunächst eher in Absichtserklä-

125 Ebenda, S. 4641.

$126 \mathrm{JO}$, Chambre des Députés, Débats parlementaires, $2^{\mathrm{e}}$ séance du 24 décembre 1929, S. 4643.

127 Ebenda.

128 Diesen Gedanken entfaltet als ein Resümee der Phase der „Locarno-Politik“: Wurm, Sicherheitspolitik, S. 555. 
rungen und im Atmosphärischen zu greifen, lag hier auch der Prioritätenwechsel, den die Regierung Tardieu im Verhältnis zu Deutschland einleitete. ${ }^{129}$

Die Rede Paul Reynauds vom 24. Dezember 1929 bildet die erste greifbare Schnittstelle zwischen der Entwicklung seiner persönlichen deutschlandpolitischen Vorstellungen und den Präferenzen des neuen Ministerpräsidenten, wie dieser sie in seiner Regierungserklärung vom 7. November öffentlich formuliert hatte. Dessen Absicht, bei aller Fortsetzung der laufenden Verhandlungen den deutschen Revisionsbemühungen eine Position der "fermeté" entgegenzusetzen, fand in den Äußerungen Reynauds gewissermaßen ihre auf mittlerer Abstraktionsebene gehaltene Ausformulierung. 130

Tardieus Neuorientierung der französischen Politik gegenüber Deutschland beabsichtigte keineswegs, im Sinne einer programmatischen Kehrtwende an den Grundlagen des beiderseitigen Verhältnisses zu rühren, wie sie sich seit Locarno und den damit verbundenen Folgeverhandlungen eingestellt hatten. Realistisch genug hatte er mit der Person Briands nicht nur den wichtigsten französischen Exponenten dieser Politik in sein Kabinett übernommen, sondern bekannte sich in überzeugender Weise als „Parteigänger der Briandschen Außenpolitik“131. Die wichtigsten Fixpunkte der französischen Haltung - so die Durchführung des Young-Plans, die Räumung des Rheinlands, die Fortsetzung der wirtschaftlichen Ausgleichsbemühungen, die Unnachgiebigkeit in Entwaffnungsfragen und das prinzipielle Festhalten am Versailler Vertrag - blieben unangetastet. ${ }^{132}$ Die atmosphärische Veränderung, die Tardieu verantwortete, rührte vielmehr von der bereits auf der zweiten Haager Konferenz im Januar 1930 ins Werk gesetzten Absicht zur "Nachbesserung “ ${ }^{133}$ schwebender Verhandlungsgegenstände im Interesse Frankreichs. Der neue, energische Führungsstil Tardieus ließ auch die Prärogativen seines Außenministers nicht ungeschmälert: „Im Verhältnis zu Poincaré konnte Briand mit Recht im vertraulichen Gespräch sagen, daß er diesen nach und nach zu seiner politischen Auffassung bekehrt habe und daß der Schüler gute

129 Die konzeptionellen Grundlagen und die Ziele der Deutschlandpolitik André Tardieus wurden bisher noch nicht grundlegend untersucht. Die neueste umfassende Darstellung der politischen Laufbahn des Politikers - François Monnet, Refaire la République. André Tardieu, une dérive réactionnaire (1876-1945), Paris 1993 - konzentriert sich auf den Staatsreformer und politischen Denker und geht etwa auf seine Deutschlandpolitik nicht ein. Auch in älteren Arbeiten spielt dieser wichtige Aspekt keine Rolle: Rudolph Binion, Defeated Leaders. The political fate of Caillaux, Jouvenel and Tardieu, New York 1960; L. Aubert/I. Martin/M. Missoffe/F. Piétri/A. Pose, André Tardieu, Paris 1957.

Wichtige Hinweise im Rahmen eines umfassenderen Ansatzes bietet Knipping, Ende der LocarnoÄra, S. $95 \mathrm{ff}$.; zu nennen auch Hagspiel, Verständigung, S. $467 \mathrm{f}$. Aufschlußreich ist auch die Charakteristik der Neuakzentuierung in der französischen Deutschlandpolitik durch den deutschen Botschafters in Paris: „Die Idee der Versöhnung als leitender idealer Zweck ist mehr in den Hintergrund getreten gegenüber Gedanken der Verfolgung egoistischer nationaler Ziele, zu deren Erreichung u. a. auch die Wiederversöhnung dienen soll." (Hoesch an Auswärtiges Amt, 29. 12. 1929; ADAP B XIII, Nr. 228, S. 477-480, hier: S. 479).

130 Vgl. hierzu die Regierungserklärung Tardieus und seine Debattenstellungnahme vom 9. November in: Le Temps, 8.11. 1929 (Zitat) und 10.11. 1929. Zum Regierungsprogramm Tardieus vgl. ausführlich das folgende Kapitel dieser Arbeit.

131 So in seinem ersten Gespräch mit dem deutschen Botschafter: Hoesch an AA, 14. 11. 1929 (ADAP B XIII, Nr. 124, S. 265-268, hier: S. 266).

132 Dazu auch der Überblicksbericht Hoeschs: Hoesch an AA, 29. 12. 1929 (ADAP B XIII, Nr. 228, S. $477-480$, bes. S. 479 ).

133 Knipping, Ende der Locarno-Ära, S. 95. 
Fortschritte mache und manchmal seinen Lehrer selbst erstaune. Im Falle Tardieu, der sich wohl auch an Briand herangebildet hat, ist der Schüler der Schule längst entwachsen und formt und knetet eigenmächtig das Gebilde des Lehrers, der seinerseits der neuen Formgebung etwas mißmutig sich anbequemt. " 134

Auch Reynaud war nun gewillt, den für Deutschland aus den internationalen Implikationen der deutsch-französischen Annäherungspolitik erwachsenen Spielraum zur Umgestaltung des Versailler Systems zu begrenzen. Das zumindest geht aus der Nachdrücklichkeit hervor, mit der er die Einbettung des reinen Verständigungsansatzes in ein mehrschichtiges sicherheitspolitisches Konzept propagierte. Ziel der französischen Politik müsse es sein, die andere Seite an der gewaltsamen Wiederherstellung der Vorkriegssituation zu hindern. Ingesamt entwarf Reynaud in der Kammerdebatte von Ende Dezember 1929 und in einer Folge von Artikeln in der politischen Presse ${ }^{135}$ eine außenpolitische "Doppelstrategie“ von Gesprächsbereitschaft und Widerstandsbeharren. Sie sollte auf der Basis der strukturellen Erneuerung und Stärkung des französischen Potentials unter antikommunistischem und "kapitalistischem" Vorzeichen die Rückkehr Frankreichs in eine zumindest moralische Führungsrolle in der Welt ermöglichen. Nicht ohne durch die nationalistische, systemkritische Rechte einer allzu eilfertigen Verzichtspolitik gegenüber Deutschland beschuldigt zu werden, faßte er diese Ideen unter dem Leitbegriff des „Néo-nationalisme“ zusammen. 136

„Seuls, les peuples forts et calmes peuvent utilement travailler pour la paix." 137 _ Diese Devise Tardieus bestimmte bereits die politische Zusammenarbeit mit Paul Reynaud, schon bevor dessen Berufung ins Finanzministerium Anfang März 1930 die Kooperation institutionalisierte. Sie strukturierte später die gemeinsamen argumentativen Anstrengungen im Umfeld der Ratifizierung der Haager Beschlüsse in Frankreich; sie prägte auch die politische Instrumentalisierung der finanzpolitischen Präponderanz Frankreichs als Mittel zur Eindämmung deutscher Revisi-

134 In üblicher Prägnanz und Gedankenschärfe: Hoesch an AA, 29. 12. 1929 (ADAP B XIII, Nr. 228, S. 478). Zum Verhältnis Tardieu-Briand in bezug auf die Fortsetzung der Politik gegenüber Deutschland: Knipping, Ende der Locarno-Ära, S. 94-96; Hagspiel, Verständigung, S. 467 f.; allgemein auch Bonnefous, Histoire IV, S. 375-379. Zu stark harmonisiert das Einverständnis der beiden Politiker: Siebert, Aristide Briand, S. 523-526.

$135 \mathrm{JO}$, Chambre des Députés, Débats parlementaires, $2^{\mathrm{c}}$ séance du 24 décembre 1929, S. $4642 \mathrm{f}$. Vgl. dazu die mehrwöchige publizistische Auseinandersetzung Reynauds mit dem rechtsstehenden Herausgeber der Revue Hebdomadaire, François Le Grix, in deren Rahmen er seine bereits im Dezember vorgetragenen Gedanken wiederholte und systematisierte. Zur Kontroverse im einzelnen: François Le Grix, "Le blanc-seing pour La Haye“, in: Revue Hebdomadaire, 4. 1. 1930, S. 102-120; Paul Reynaud, „Un néo-nationalisme“, Ebenda, 25.1. 1930, S. 487-492; François Le Grix, "Le patriotisme de la continuité", Ebenda, 1.2. 1930, S. 82-96; Paul Reynaud, „Le néonationalisme et la continuité “, Ebenda, 15. 2. 1930, S. 363-369; François Le Grix, „La continuité... des chutes ministérielles", Ebenda, 22. 2. 1930, S. 495-498.

136 Siehe dazu insbesondere Reynauds Ausführungen in der Revue Hebdomadaire vom 25. 1. und 15. 2. 1930. Zu den innenpolitischen Implikationen des außenpolitischen Programms speziell: „Puisque le destin de la France demeure, malgré tout, de vivre dangereusement, il faut soumettre la nation à un entêtement moral et, par exemple, écarter des enfants les maîtres communistes qui se refusent même à leur apprendre l'histoire de leur patrie. [...] Nous n'assurons la sécurité de la France qu'en travaillant à sa grandeur. Elle perdra sa situation privilégiée si son potentiel baisse, ou simplement n'augmente pas aussi rapidement que celui des nations concurrentes. Comment développer ce potentiel? Ferons-nous appel au capitalisme ou au marxisme? Nous avons choisi." (Reynaud, Un néo-nationalisme, S. $490 \mathrm{f}$.).

137 So geäußert in Tardieus Regierungserklärung vom 7. November 1929 (Le Temps, 8. 11. 1929). 
onsbestrebungen ab Sommer 1930.138 Sie motivierte schließlich die geplante Einführung eines umfassenden nationalen Modernisierungsprogramms zur Erneuerung der französischen Binnenstrukturen. ${ }^{139}$

Zur Jahreswende 1929/30 stand Paul Reynaud nurmehr eine Handbreit von der politischen Macht entfernt. Seit seinem gelungenen parlamentarischen Coup vom Sommer 1929 und dem Aufstieg in den Kreis der tonangebenden Führungsriege in der „Chambre des Députés“ gehörte er zu jenen für ein Regierungsamt in Frage kommenden „ministrables“, die bei Regierungsneubildungen nicht mehr ohne weiteres übergangen werden konnten. Bereits anläßlich der Konstituierung der elften und letzten Regierung Briand im Juli 1929 war er für den zu schaffenden Posten des „Sous-secrétaire d'Etat“ im Außenministerium ins Auge gefaßt worden ${ }^{140}$; dann aber war er aufgrund der unveränderten Überführung der Regierung Poincaré in ein „Kabinett Briand ohne Poincare“"141 nicht zum Zuge gekommen. Als dann im November 1929 André Tardieu seine erste Mitte-Rechts-Regierung bildete, in der unter anderem Reynauds Parteikollegen Flandin und FrançoisPoncet Minister- und Staatssekretärsposten erhielten, wäre seine Berufung nicht opportun gewesen. Angesichts der kaum verebbten landesweiten Pressekampagne gegen ihn hätte er zweifellos eine schwere Belastung für das neue Kabinett dargestellt, das ohnehin durch die fehlende Partizipation der Radikalsozialisten gefährdet war. Mehr und mehr entwickelte sich Reynaud dann in der Folge aber auch außerhalb der Regierungsverantwortung zu einer tragfähigen Stütze Tardieus in der Kammer der Abgeordneten. Und wenn die geleistete Hilfestellung wie im Falle der deutschlandpolitischen Debatte vom Jahresende 1929 sicherlich weitestgehend in sachlicher Übereinstimmung begründet war, so umfaßte sie daneben eine parlamentstaktische Komponente. Hinter ihr stand nicht zuletzt die Absicht Reynauds, sich durch erfolgreiche Zusammenarbeit für ein Ministeramt zu empfehlen.

In der Tat hatten ja seine Ausführungen vom Dezember 1929 aufgrund der innerparlamentarischen Konnotationen, die die Debatte befrachteten, zusätzliches Gewicht gewonnen. Im Oktober 1929 war Briand bekanntlich weniger aufgrund der Inhalte seiner Den Haager Verhandlungsführung als der praktizierten restriktiven Informationspolitik gegenüber der Kammer vorwiegend mit den Stimmen der sozialistischen und radikalsozialistischen Linken gestürzt worden. ${ }^{142}$ André Tardieu hatte auf außenpolitischem Gebiet nicht nur die Fortsetzung der laufen-

138 Vgl. die Stellungnahmen Reynauds während der Ratifikationsdebatte: JO, Chambre des Députés, Débats parlementaires, 2 e séance du 28 mars 1930, S. 1375-1382 sowie die Debatten des Folgetags: ebenda, $2^{e}$ séance du 29 mars 1930, S. $1408 \mathrm{ff}$.; siehe dazu auch den gemeinsamen Auftritt vor der Finanzkommission der Kammer: Archives de l'Assemblée Nationale, Commission des Finances. Procès-verbal de la $3^{\mathrm{e}}$ séance du 21 mars 1930; ebenda, Procès-verbal de la $2^{\mathrm{e}}$ et $3^{\mathrm{e}}$ séance $\mathrm{du}$ 22 mars 1930.

$\mathrm{Zu}$ den komplexen politischen, finanz- und reparationstechnischen Zusammenhängen umfassend und auf breiter empirischer Basis: Philipp Heyde, Das Ende der Reparationen. Deutschland, Frankreich und der Youngplan 1929-1932, Paderborn u.a. 1998, S. 35 ff.; Knipping, Ende der Locarno-Ära, S. 168-175; 199-202.

139 Vgl. Kapitel VIII.2 dieser Arbeit.

140 Seine Beinahe-Berufung kursierte als "On-dit“ im politisch-publizistischen Milieu: vgl. etwa L'Humanité, 23. 9. 1929.

141 Siebert, Aristide Briand, S. 483.

$1+2$ Ebenda, S. 521 f.; Bonnefous, Histoire IV, S. 367-369. 
den Verhandlungen angekündigt, sondern sich demonstrativ hinter seinen Außenminister Briand gestellt, als dieser vom rechten Kammerflügel zur Revision seiner Politik des Entgegenkommens gegenüber Deutschland aufgefordert worden war. Eine Klärung der Mehrheitsverhältnisse war nun angesichts der wochenlang äußerst unklaren Situation dringend nötig. Die Mitte-Rechts-Mehrheit der Kammer, auf die sich Tardieu in erster Linie stützte, trug die Fortführung der Locarno-Politik eher aus Loyalität zum Regierungschef denn aus Überzeugung mit: Bereits Anfang November hatte es einer „oratorischen Meisterleistung" Briands bedurft, damit das Kabinett nicht über die Deutschlandfrage zu Fall kam. Dagegen ging die sozialistische und die bürgerliche Linke aus Mißtrauen gegenüber dem neuen Regierungschef auf Distanz zu einer Politik, die im Prinzip die eigene war. ${ }^{143}$ Vor dieser Konstellation zielten Reynauds Intervention und ein von ihm eingebrachter, sachlich kaum motivierter Antrag zur Reduzierung des Budgets des Außenministeriums auf eine letzte Klärung der Geister und der parlamentarischen Fronten am Vorabend der zweiten Konferenz von Den Haag. ${ }^{144}$ Das Manöver gelang: Gestützt auf ein „überraschend großes Vertrauensvotum “ 145 beider Kammern, die sich am 20. bzw. 27. Dezember $1929 \mathrm{klar}$ für das Programm Tardieus aussprachen, konnte der Ministerpräsident Anfang Januar 1930 in die niederländische Hauptstadt aufbrechen. ${ }^{146} \mathrm{Zu}$ seiner Delegation gehörte auch der Abgeordnete Paul Reynaud. ${ }^{147}$

Der „Lohn“ für Reynauds Loyalität kam, nachdem Tardieus erste Regierung über der Unnachgiebigkeit ihres Finanzministers Chéron in Budgetfragen Mitte Februar 1930 zu Fall gekommen war.

${ }_{143} \mathrm{Zu}$ den Schwierigkeiten Tardieus und Briands, dem Kabinett die Unterstützung der eigenen MitteRechts-Mehrheit für die anvisierte Deutschlandpolitik zu verschaffen, nachdem die Radikalsozialisten und selbst die Gruppierung Briands, die „Républicains socialistes“, sich von der als zu weit rechtsstehend eingestuften Regierungsformation distanziert hatten vgl. die Berichte des deutschen Botschafters vom 7. und 9. November sowie vom 29. Dezember 1929 (Hoesch an AA, 7. 11. 1929, ADAP B XIII, Nr. 107, S. 232 f.; Hoesch an AA, 9. 11. 1929, Ebenda, Nr. 113, S. 245-249 (Zitat S. 245); Hoesch an AA, 29. 12. 1929, Ebenda, Nr. 228, S. 477-480, hier: S. 477).

144 Vgl. dazu die aufschlußreiche Analyse von François Le Grix, „Le blanc-seing pour La Haye“, in: Revue Hebdomadaire, 4. 1. 1930. Ein Hinweis auf Reynauds Absichten auch in: Reynaud, Mémoires I, S. 286. Zu seinem Antrag: JO, Chambre des Députés, Débats parlementaires, $2^{\mathrm{e}}$ séance du 27 décembre 1929.

145 So Botschafter von Hoesch an das Auswärtige Amt, 29. 12. 1929 (ADAP B XIII, Nr. 228, S. 477).

146 Zum Ausgang der entscheidenden Abstimmungen in Chambre des Députés und Senat: Bonnefous, Histoire IV, S. 380 f.

147 Dies nach eigener Aussage Tardieus anläßlich seiner Anhörung zum Youngplan vor den gemeinsam tagenden Ausschüssen für Finanzen und Auswärtiges der Kammer der Abgeordneten am 21. März 1930 (Archives de l'Assemblée Nationale, Commission des Finances, Procès-verbal de la 3e séance du 21 mars 1930). 